\title{
Dynamic mesh adaptation for front evolution using discontinuous Galerkin based weighted condition number relaxation
}

\author{
Patrick T. Greene*, Samuel P. Schofield, Robert Nourgaliev \\ Lawrence Livermore National Laboratory, Design Physics Division, Livermore, CA, 94551, USA
}

\begin{abstract}
A new mesh smoothing method designed to cluster cells near a dynamically evolving interface is presented. The method is based on weighted condition number mesh relaxation with the weight function computed from a level set representation of the interface. The weight function is expressed as a Taylor series based discontinuous Galerkin projection, which makes the computation of the derivatives of the weight function needed during the condition number optimization process a trivial matter. For cases when a level set is not available, a fast method for generating a low-order level set from discrete cell-centered fields, such as a volume fraction or index function, is provided. Results show that the low-order level set works equally well as the actual level set for mesh smoothing. Meshes generated for a number of interface geometries are presented, including cases with multiple level sets. Dynamic cases with moving interfaces show the new method is capable of maintaining a desired resolution near the interface with an acceptable number of relaxation iterations per time step, which demonstrates the method's potential to be used as a mesh relaxer for arbitrary Lagrangian Eulerian (ALE) methods.
\end{abstract}

Keywords: Weighted mesh smoothing, Condition number mesh relaxation, r-refinement, Level set, Discontinuous Galerkin discretization, ALE method, WENO

\section{Introduction}

Mesh relaxation and adaptation is used to improve mesh quality and, ultimately, solution accuracy. This arises in static and dynamic applications. In static applications, a relaxation algorithm is applied to an initial mesh to improve its quality and may be used to concentrate mesh zoning in areas such as multi-material interfaces, or boundary layers, which are likely to require enhanced accuracy to resolve local length scales. In dynamic applications, in particular in arbitrary Lagrangian Eulerian (ALE) [1] methods, the mesh motion may differ from the material motion. Analysts might want to prescribe objectives for the mesh motion, such as proximity to the Lagrangian mesh, improved element quality, and generating finer zoning in regions of

\footnotetext{
* Corresponding author

Email address: greene30@llnl.gov (Patrick T. Greene) 
interest [2]. This work develops an innovative algorithm for utilizing condition number mesh relaxation to improve mesh quality while also enhancing mesh resolution at interfaces or at any location as prescribed with discrete weights.

There are several variants of mesh relaxers that have been developed in the past. The most widespread method is Equipotential or Winslow mesh smoothing [3]. In Winslow smoothing, physical mesh line positions are regarded as a function of a parametric variable and the gradient of the mesh line positions with respect to that parametric coordinate are minimized in an iterative procedure. A weight can be naturally incorporated that effectively scales the Jacobian of the zone and draws mesh into higher weight regions according to the ratio of weights. However, due to the global behavior of the relaxer, Winslow smoothing can significantly disrupt local mesh regions that may be very regular, therefore degrading the local mesh quality.

An increasingly popular alternative method is the condition number mesh relaxation method developed by Knupp $[4,5,6]$. The method defines a condition number functional at each vertex that is a function of the geometry of all the cells that share that vertex ${ }^{1}$. By minimizing the condition number at all the vertices, the overall quality of the mesh increases. The inclusion of a barrier function in the objective functional ensures that the method cannot produce an invalid mesh from an initially valid mesh, making the method extremely robust. However, the original method did not provide a process for the flow physics to influence the mesh relaxation process. Knupp et al. [2] addressed this issue with their reference Jacobian method. The method would use the Lagrangian motion of the mesh to compute a reference Jacobian, which could then be used to constrain the mesh relaxation.

Váchal and Maire [7] extended the original work of Knupp and derived a weighted condition number mesh relaxation method for general unstructured computational meshes. Similar to the work of Knupp et al., their goal was to provide a link between the physics of the problem and the mesh relaxation. This was achieved by the introduction of a weight term in the condition number functional. By varying the value of the weight, different cell sizes could be obtained. If the weights are computed from flow variables, this provides a method for clustering mesh cells near areas of interest. A focus of their work is the proper discretization of the weighted condition number functional and they mention a number of methods for computing the derivatives of the weight function needed in the optimization process. Although methods for discrete derivatives were discussed, all the results shown were computed using analytical functions and their derivatives. Extensions to use discretely defined weights, for instance, piecewise constant zone centered data, have not been demonstrated in general due to difficulties in reconstructing sufficiently smooth $\left(C^{2}\right)$ representations of the original discontinuous weights [8].

In this paper, we present a new mesh relaxation method that is designed to cluster cells near moving

\footnotetext{
${ }^{1}$ Knupp originally defined a family of mesh relaxation methods distinguished by the choice of matrix norm associated with the element Jacobian. Here, we use the term condition number relaxation to denote Knupp's method as prescribed using the Frobenius norm. This is the most widely used and recommended version.
} 
fronts and interfaces. The method is based on the weighted condition number relaxation of Váchal and Maire. The discretization issues discussed by Váchal and Maire are resolved by using a discontinuous Galerkin representation for the weight function. This allows the spatial derivatives of the weight function to be easily computed since the spatial dependency of the discontinuous Galerkin projection only comes from the prescribed basis functions, which have a known analytic form. The method can adapt to dynamic fronts by having the weight be a function of a level set representation of the front. Level set functions provide a method for determining the shortest distance to the interface from any point in the mesh, which can be used to adjust the amplitude of the condition number weight based on its proximity to the interface. This dependency between the weight and the distance is specified through a mapping function, which can be used to adjust the location and size of refinement zones. For cases when a level set function of the front is not available, we provide a method for generating a low-order level set from a discrete cell-center field. This low-order level set can then be used to create a weight function that generates meshes very similar to the actual level set.

The new mesh relaxation method presented in this work can be divided into two parts: the discontinuous Galerkin discretization of the weighted condition number relaxation method and the procedure for computing a weight function from a given level set representation of an interface. Section 2 of this manuscript provides an overview of weighted condition number relaxation and presents the new discontinuous Galerkin weights. The generation of the weights from an interface's level set function is discussed in Section 3. Also included is the method for generating a low-order level set from discrete cell-centered fields. Results for numerical examples of static interfaces are shown in Section 4. These examples represent the generation of a mesh for purely Eulerian simulations or the initial mesh for an ALE simulation. This is followed by results for dynamic interfaces in Section 5, which are intended to demonstrate the potential of the new relaxation method for ALE simulations. The paper finishes with the conclusions in Section 6 .

\section{Discontinuous Galerkin weighted condition number mesh relaxation}

\subsection{Weighted condition number mesh relaxation}

Condition number $(\mathrm{CN})$ relaxation works by defining a functional at each vertex of a mesh that represents the quality of the neighboring cells and then minimizing the functionals for the entire mesh, which should improve the overall quality of the mesh. When applied to a quadrilateral mesh, the original formulation of the $\mathrm{CN}$ relaxation method is designed to create cells with orthogonal edge vectors and unit aspect ratios. The inclusion of a weight function permits the clustering of mesh cells in areas of interest. The local functional is minimized using Newton's method in a Jacobi relaxation fashion. That is to say, a new vertex position is computed by minimizing the local functional with all the neighboring vertices fixed. Once the new positions for all the vertices are computed, the entire mesh is updated at once. The process of applying a single step 


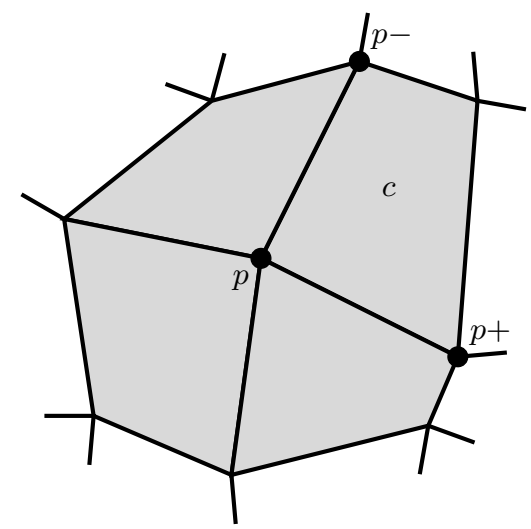

Figure 1: Sample mesh showing the cells affected by the minimization of the condition number for vertex $p$. The vertices neighboring $p$ in the clockwise $(p-)$ and counterclockwise $(p+)$ directions for the cell labeled $c$ are also shown.

of Newton's method to each vertex and then updating all the vertex positions will be denoted as a single iteration of the mesh relaxation method.

Figure 1 shows a sample mesh around vertex $p$. In this work, we only consider quadrilateral cells, but the method can be extended to arbitrary cells. In addition, for simplicity, only two-dimensional meshes are discussed. The extension of the method to three-dimensional meshes is straightforward. All the cells affected by the movement of point $p$ are shown in gray. Within a given cell, the vertex connected to $p$ in the clockwise direction is labeled $p-$. Similarly, the counterclockwise vertex is labeled $p+$. Váchal and Maire [7] defined the local discrete $\mathrm{CN}$ functional for vertex $p$ as:

$$
F\left(\mathbf{x}_{p}\right)=\sum_{c \in \mathcal{C}(p)}\left(W^{c p} \frac{M^{c p}}{J^{c p}}+W^{c p-} \frac{M^{c p-}}{J^{c p-}}+W^{c p+} \frac{M^{c p+}}{J^{c p+}}\right)
$$

where $\mathbf{x}_{p}=\left(x_{p}, y_{p}\right)$ is the position of vertex $p$ and $\mathcal{C}(p)$ is the subset of all the cells that share $p$ as a vertex. If $q$ represents one of the vertices in the set $\{p, p+, p-\}$, then $W^{c q}$ is the weight function computed in cell $c$ at vertex $q$, and $M^{c q}$ and $J^{c q}$ are geometric functions computed from the edge vectors at vertex $q$ of cell c. The geometric functions are defined as

$$
M^{c q}=\left\|\mathbf{e}_{c q}^{+}\right\|^{2}+\left\|\mathbf{e}_{c q}^{-}\right\|^{2} \quad \text { and } \quad J^{c q}=\left\|\mathbf{e}_{c q}^{+} \times \mathbf{e}_{c q}^{-}\right\|
$$

where $\|\cdot\|$ is the $L_{2}$ norm. The vectors $\mathbf{e}_{c q}^{+}$and $\mathbf{e}_{c q}^{-}$are the edge vectors starting at vertex $q$ and pointing to the next vertex of cell $c$ in the counterclockwise and clockwise direction, respectively. Figure 2 shows all the edge vectors in cell $c$ from Figure 1 used to calculate the functional at vertex $p$.

The local discrete functional is minimized using Newton's method. This is equivalent to solving the coupled equations

$$
\frac{\partial F}{\partial x_{p}}\left(\mathbf{x}_{p}\right)=0 \quad \text { and } \quad \frac{\partial F}{\partial y_{p}}\left(\mathbf{x}_{p}\right)=0
$$




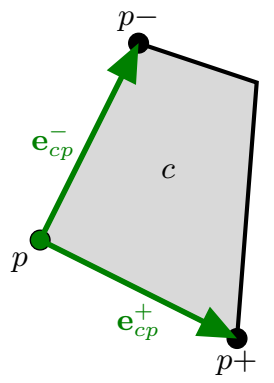

(a) Edge vectors for point $p$.

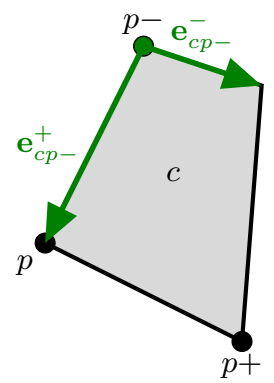

(b) Edge vectors for point $p-$.

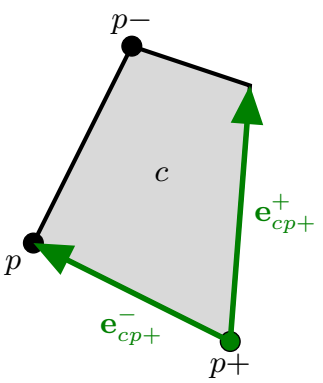

(c) Edge vectors for point $p+$.

Figure 2: Edge vectors in cell $c$ from Figure 1 used in the calculation of the functional for vertex $p$.

using the two-dimensional Newton-Raphson iterative algorithm. Solving these equations will require the first and second derivatives of $M^{c q}, J^{c q}$, and $W^{c q}$, with respect to $x_{p}$ and $y_{p}$. The calculation of $M^{c q}, J^{c q}$, and their derivatives is straightforward since they only involve the edge vectors, which have an analytic form. Computing $W^{c q}$ and its derivatives can be difficult as the weight may be a function of discrete values from the physics solver. For the Newton based minimization procedure, a reconstruction or smoothing step must be applied to the weights to obtain a sufficiently smooth approximation for the first and second derivatives of the weight function to be meaningful.

\subsection{Discontinuous Galerkin weights}

To create a twice differentiable representation of the weights within each cell, a piecewise-quadratic DG projection of the weight function is used. A piecewise-quadratic projection was chosen since it is the simplest projection with non-zero second derivatives. The projection allows the weight function within cell $c$ to be expressed as

$$
W^{c}(\mathbf{x})=\sum_{n=1}^{N} W_{n}^{c} b_{n}^{c}(\mathbf{x})
$$

where $W_{n}^{c}$ is the $n$th degree of freedom in cell $c, b_{n}^{c}$ is the $n$th basis function in cell $c$, and $N$ is the total number of degrees of freedom for the projection. In the present work, we use the modal DG formulation with basis functions derived from a Taylor series expansion around the centroid of the cell [9]. For a twodimensional piecewise-quadratic projection, there are a total of six degrees of freedom with the following basis functions.

$$
\begin{aligned}
& b_{1}^{c}=1, \quad b_{2}^{c}=\bar{x}, \quad b_{3}^{c}=\bar{y}, \\
& b_{4}^{c}=\frac{\bar{x}^{2}}{2}-\frac{1}{2 \Omega_{c}} \int_{\Omega_{c}} \bar{x}^{2} d \Omega, \quad b_{5}^{c}=\bar{x} \bar{y}-\frac{1}{\Omega_{c}} \int_{\Omega_{c}} \bar{x} \bar{y} d \Omega, \quad b_{6}^{c}=\frac{\bar{y}^{2}}{2}-\frac{1}{2 \Omega_{c}} \int_{\Omega_{c}} \bar{y}^{2} d \Omega,
\end{aligned}
$$

where

$$
\bar{x}=\frac{x-x_{c}}{\Delta x}, \quad \bar{y}=\frac{y-y_{c}}{\Delta y}, \quad \Delta x=\frac{1}{2}\left(x_{\max }-x_{\min }\right), \quad \Delta y=\frac{1}{2}\left(y_{\max }-y_{\min }\right),
$$


$\left(x_{c}, y_{c}\right)$ is the centroid of the cell, $\Omega_{c}$ is the area of the cell, and $x_{\max }, x_{\min }, y_{\max }$ and $y_{\min }$ are the maximum and minimum nodal coordinates of the cell in the $x$ - and $y$-direction, respectively. Note, the projected weights are discontinuous at element boundaries.

When computing the quantity $W^{c q}$, we follow the advice of Váchal and Maire and use an average of the weight function evaluated at vertex $q$ and its two neighbors $(q+$ and $q-)$,

$$
W^{c q}=\frac{1}{3}\left(W^{c}\left(\mathbf{x}_{q}\right)+W^{c}\left(\mathbf{x}_{q+}\right)+W^{c}\left(\mathbf{x}_{q-}\right)\right) .
$$

By using a DG projection for the weights, the calculation of its derivatives becomes trivial, since they now only involve derivatives of the known basis functions. For example, the derivative of $W^{c p}$ with respect to $x_{p}$ can be expressed as

$$
\frac{\partial W^{c p}}{\partial x_{p}}=\frac{1}{3} \frac{\partial}{\partial x_{p}}\left(W^{c}\left(\mathbf{x}_{p}\right)+W^{c}\left(\mathbf{x}_{p+}\right)+W^{c}\left(\mathbf{x}_{p-}\right)\right) .
$$

In this expression, only the first term, $W^{c}\left(\mathbf{x}_{p}\right)$, is a function of $\mathbf{x}_{p}$. Therefore, the derivative of the second and third terms, $W^{c}\left(\mathbf{x}_{p+}\right)$ and $W^{c}\left(\mathbf{x}_{p-}\right)$, are simply zero and Equation (8) simplifies to

$$
\frac{\partial W^{c p}}{\partial x_{p}}=\frac{1}{3} \frac{\partial W^{c}}{\partial x_{p}}\left(\mathbf{x}_{p}\right) .
$$

Substituting in the DG projection, the expression for the derivative becomes

$$
\frac{\partial W^{c p}}{\partial x_{p}}=\frac{1}{3} \sum_{n=1}^{N} W_{n}^{c} \frac{\partial b_{n}^{c}}{\partial x_{p}}\left(\mathbf{x}_{p}\right) .
$$

To permit the mesh to adapt to moving interfaces, the weight function will be computed from the signed distance function of a level set used to represent the interface. On its own, this can cause issues with the mesh because the signed distance function may only be $C^{0}$ continuous at some locations. If a discontinuity in the slope of the sign distance function is located within a cell, then the DG projection of the weight function can be ill behaved and lead to extreme stretching of the mesh. To address this, the degrees of freedom for the projection of the weights are computed using the weighted essentially nonoscillatory (WENO) reconstruction of Luo et al. [10, 11]. The WENO reconstruction provides a method for limiting the quadratic degrees of freedom and thus alleviating the issues caused by the discontinuities. WENO limiters are preferable for a Newton's method based algorithm because the solution will remain differentiable through the Newton iterations. Other limiters usually contain conditional statements in their algorithm. If a solution is near the conditional statement switch, Newton's method may oscillate between the two states and prevent convergence.

The WENO reconstruction of Luo et al. can be divided into three steps. First, the degrees of freedom for the constant and linear basis functions are computed using an $L_{2}$ projection of the weight function onto the basis functions. The resulting degrees of freedom provide a linear projection of the weight function, which is usually denoted as $\mathrm{DG}\left(\mathrm{P}_{1}\right)$ in the literature. The second step of the method is to compute a least-squares 
reconstruction of the quadratic degrees of freedom from the constant and linear degrees of freedom in the current cell and its face neighbors. This provides a piecewise-quadratic projection of the weight function and the reconstructed projection is expressed as $\operatorname{rDG}\left(\mathrm{P}_{1} \mathrm{P}_{2}\right)$ in the literature $[12,13,10,11]$. This projection will be ill behaved if any discontinuities are present in the cell. To rectify this, the final step of the WENO reconstruction is to apply a limiter to the quadratic degrees of freedom. The limiter is simply a weighted average of the degrees of freedom computed during the reconstruction. This will result in a new piecewisequadratic projection that can then be used to compute the weights for the $\mathrm{CN}$ functional. In addition to the work of Luo et al., details about the reconstruction can be found in Greene et al. [14].

The WENO reconstruction helps reduce the effect of any discontinuities found within a cell. Discontinuities, however, still exist at cell interfaces. This comes from the fact that the DG projection does not enforce continuity at the cell interfaces. This can result in different values being computed for a given vertex when projections from different cells sharing that vertex are used. In our work, there were no noticeable issues caused by these discontinuities and therefore no special treatment was used to correct or account for them.

\subsection{Measure of convergence}

Since weighted CN relaxation is an iterative method, a measure of convergence is required. In this work, the convergence metric $\left(\epsilon_{c}\right)$ is calculated as

$$
\epsilon_{c}=\frac{1}{N_{\mathcal{P}}} \sum_{p \in \mathcal{P}}\left(\frac{\left\|\Delta \mathbf{x}_{p}\right\|}{\sqrt{\Omega_{R}}}\right),
$$

where $\Delta \mathbf{x}_{p}$ is the change in the position of vertex $p$ during the last iteration, $\Omega_{R}$ is a reference area for the entire mesh, $\mathcal{P}$ is the set of all vertices in the computational domain, and $N_{\mathcal{P}}$ is the number of vertices in $\mathcal{P}$. For the reference area, we use the minimum cell area of the initial mesh.

\section{Level set based weight function}

With the DG discretization of the CN relaxation method covered, we now turn to the procedure for generating the value of the weights. Our new mesh adaptation method relies on level set functions to specify the interfaces requiring increased refinement. By representing the interface with a level set, specifically a signed distance function, the local magnitude of the weight can vary with the distance to the interface. A key part of the method is the distance to weight mapping function, which provides a way to bound the amplitude of the weight and control the locations of increased resolution. The mapping allows us to produce weight functions with a minimum value, which corresponds to the location of minimum cell size, at interfaces and have it extend from the interface any desired distance. 


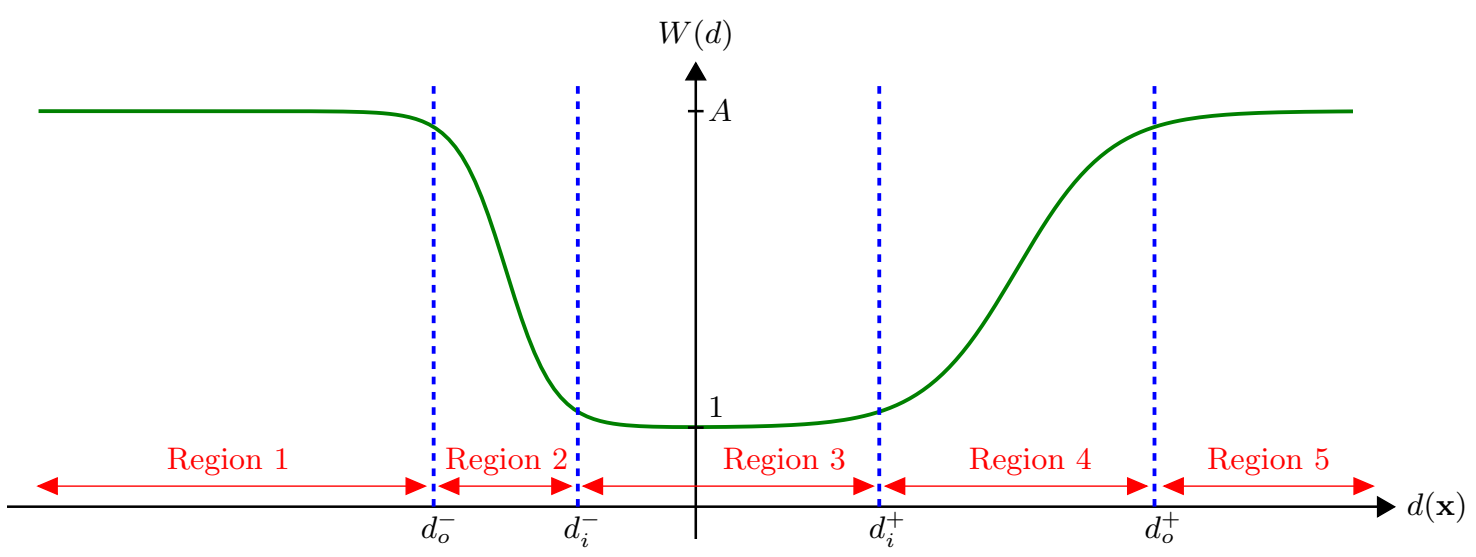

Figure 3: Qualitative plot of the "signed distance function to weight function" mapping.

\subsection{Level set function and signed distance function}

A level set is a function designed to separate a computational domain into two regions, corresponding to positive and negative values of the level set function. The interface between these two regions is defined by the zero contour of the level set function. A specific type of level set is the signed distance function $(\mathrm{SDF}), d(\mathbf{x})$. In addition to having the sign tell you which region you are in, the magnitude of the SDF is the shortest distance to the interface. This property makes the SDF an excellent choice for dynamic mesh adaptation to moving fronts. The process of going from a general level set to an SDF is called re-distancing or re-initialization and there are a few different algorithms for the procedure available [15, 16].

We use a piecewise-quadratic DG projection to represent the $\mathrm{SDF}$ in cell $c$,

$$
d^{c}(\mathbf{x})=\sum_{n=1}^{N} d_{n}^{c} b_{n}^{c}(\mathbf{x}) .
$$

The degrees of freedom can be computed from an $L_{2}$ projection of the SDF onto the basis functions. The WENO limiter is not used for the SDF projection. Since the focus of this work is on the mesh relaxation method, the DG projection for the SDF will be computed from the analytic solution. A DG based method for dynamic re-distancing on arbitrary meshes is described in Nourgaliev et al. [17].

\subsection{Signed distance function to weight function mapping}

Since differences in the magnitude of the weight function will result in differently sized mesh cells, with smaller values generating smaller cells, the weight function should have a minimum near regions of interest. To provide extra control over the location and size of the refinement area, we use the "signed distance function to weight function" mapping ${ }^{2}$ shown in Figure 3. The weight function in this figure is divided

\footnotetext{
${ }^{2}$ Instead of prescribing the weight function $W$, Váchal and Maire specified the inverse of it. We found it is simpler to control the distribution of the weight function when $W$ is prescribed directly, instead of its inverse.
} 
into five regions. Region 3 includes the interface and also encapsulates the area with the minimum of the weight function, which should result in the smallest mesh cells. Regions 1 and 5 contain the maximum of the weight function in the negative and positive directions of the SDF, respectively. This should result in the largest cells being located in these regions. Regions 2 and 4 are transitional areas, where the cell sizes will smoothly vary from the fine mesh in region 3 to the coarse mesh in regions 1 and 5 , respectively.

The weight function mapping from Figure 3 can be expressed as

$$
W(d)=\frac{A-1}{2}\left[\tanh \left(\left(d-d_{m}^{+}\right) S^{+}\right)-\tanh \left(\left(d-d_{m}^{-}\right) S^{-}\right)\right]+A,
$$

where

$$
d_{m}^{ \pm}=\frac{d_{i}^{ \pm}+d_{o}^{ \pm}}{2} \quad \text { and } \quad S^{ \pm}=2 \frac{\tanh ^{-1}(\zeta)}{\left|d_{i}^{ \pm}-d_{o}^{ \pm}\right|} .
$$

$A$ is the weight function amplitude and it controls the amount of refinement enforced in region 3 . The parameter $\zeta$ specifies how much of the change from $A$ to 1 is located between $d_{o}^{ \pm}$and $d_{i}^{ \pm}$, there by providing a method for controlling the rate of change of the weight function. A value of 0.9 was used for $\zeta$ in this work.

The mapping defined by Equation (13) provides far more control over the weight function distribution than what is traditionally available in weighted Winslow smoothing. In Winslow smoothing, the weights are used as a scaling for the Jacobian of a cell and the value is usually defined by a material property. This results in roughly uniform cells on each side of a material interface, with the ratio of their characteristic lengths directly proportional to the ratio of their weights. The mapping used in this work provides more options than this simple binary distribution. Although we focus on refining the mesh near interfaces, the values of $d_{o}^{ \pm}$and $d_{i}^{ \pm}$can be adjusted to provide refinement at some distance away from the interface. The values can also be altered to mimic the material property distribution used in Winslow smoothing. For example, setting $d_{o}^{-}$and $d_{i}^{-}$to very large negative values will provide an approximately uniform distribution in the material defined by the negative value of the SDF. The values of $d_{i}^{+}$and $d_{o}^{+}$can then be adjusted to provide a smooth variation of mesh resolution away from the interface. This approach is used in the examples involving small and thin objects, such as the cruciform in Section 4.2.

\subsection{Low-order signed distance function}

The mapping given by Equation (13) requires the interface to be described as an SDF. This can be a problem for most existing ALE codes since they do not use a level set method for interface tracking, and it may not always be feasible to add a level set method to the code. Instead of a level set function, these codes commonly use an index function to track the location of materials. An index function will have a single value for all pure cells on one side of the interface and a different value for all pure cells on the other side of the interface. The value for cells that contain a portion of the interface will be based on the volume fraction of each material in the cell. The index function would usually be stored as a discrete cell-centered quantity. 
An example of this is the volume fraction from the volume of fluid method [18]. For these cases, we have developed a method to generate a low-order signed distance function (LOSDF) from the index function, which can then be used in Equation (13).

The LOSDF is generated by first finding an approximation of the value of the SDF at the centroid of each cell. This value is then used as the first degree of freedom for a piecewise-quadratic DG reconstruction. The remaining degrees of freedom are generated from a least-squares reconstruction using the face and vertex neighbors of the cell. This reconstruction is often abbreviated $\operatorname{rDG}\left(\mathrm{P}_{0} \mathrm{P}_{2}\right)$ in the literature [11].

For the LOSDF, we assume the index function is +1 for pure cells on one side of the interface, -1 for pure cells on the other side of the interface, and an intermediate value that is linearly proportional to the material volume fraction for interface ("mix") cells. ${ }^{3}$ If $\psi^{c}$ represents the index function in cell $c$ and $V^{c}$ represents the volume fraction of the +1 material in cell $c$, the two values are related by the expression

$$
\psi^{c}=2 V^{c}-1 .
$$

The first step of the method is to compute the first degree of freedom in each of the interface cells. The value for cell $c$ can be approximated as

$$
d_{1}^{c}=\left(\frac{\psi^{c}}{2}\right)(\Delta x+\Delta y),
$$

where $\Delta x$ and $\Delta y$ are given in Equation (6). This expression will also be used to compute the first degree of freedom in any cell that has a value of +1 and a face neighbor with a value of -1 , and vice versa. With all the interface cells known, the value in the remaining cells can be computed from the expression

$$
d_{1}^{c}=\operatorname{sign}\left(\psi^{c}\right) \min _{i \in I}\left(\left|d_{1}^{i}+\operatorname{sign}\left(\psi^{c}\right) D_{c, i}\right|\right),
$$

where $D_{c, i}$ is the distance between the centroid of cell $c$ and the centroid of cell $i$, and $I$ is the set of all the interface cells. At this point, the first degree of freedom has been computed in every cell and the reconstruction can be applied to compute the remaining degrees of freedom. With the LOSDF projection known, it can be used to compute the weight function in the same manner as the exact SDF. It should be noted that while the LOSDF works well for mesh relaxation, we do not recommend using it for the re-initialization/re-distancing of a level set used to track an interface. The accuracy of the LOSDF is too low for this application. A more accurate DG re-distancing algorithm is described in Nourgaliev et al. [17].

In most level set based codes, the SDF is rarely computed over the entire computational domain. Calculations are usually restricted to a small area around the interface. This region is called the "narrow band" in the literature [19]. The size of the narrow band is usually determined by the stencil size of the employed

\footnotetext{
${ }^{3}$ This is a departure from the traditional definition of the index function, which is normally defined to have values between 0 and +1 . The limits of the index function were re-scaled to -1 and +1 to mimic the behavior of a level set function.
} 
discretization method and is measured in numbers of cells. To use the level set based weights, the SDF must be computed from at least $d_{o}^{-}$to $d_{o}^{+}$. This is an issue because the physical distance covered by the narrow band can become only a very small fraction of the distance between $d_{o}^{-}$and $d_{o}^{+}$as the mesh is refined. To address this issue, the narrow-band level set method can be combined with the LOSDF. The level set with its re-distancing method is used within the narrow band, while outside of the narrow band, the distance function is computed using Equation (17) and the $\mathrm{rDG}\left(\mathrm{P}_{0} \mathrm{P}_{2}\right)$ reconstruction.

\subsection{Controlling the minimum mesh cell area}

The level of mesh refinement is determined by the weight function amplitude, $A$, which is an input parameter to the mapping provided by Equation (13). It is usually more desirable to specify a target minimum cell area instead of the weight function amplitude. Due to the global nature of CN relaxation, it can be difficult to derive an explicit relationship between $A$ and the resulting minimum cell area, since specifying the same value of $A$ for different geometries can produce different levels of refinement. To address this issue, we now present a method for specifying a final minimum cell area, in place of the weight function amplitude.

This method is composed of two elements: a barrier function that is added to the original CN functional, and a weight amplitude adjustment procedure. The barrier function takes the form of an exponential that is designed to prevent the area of the mesh cells from becoming significantly smaller than the desired minimum area. With a sufficiently large weight amplitude, the barrier function is all that is needed to enforce a minimum cell area, however, the resulting mesh may oscillate slightly and never converge to a final position. The weight amplitude adjustment procedure is designed to prevent this undesired effect. Once the target cell area is obtained, the weight function amplitude will be reduced whenever the minimum cell area for the mesh is less than the specified value. In addition, the amplitude will be increased whenever the minimum cell area is much greater than the specified value, which will drive the mesh towards the target area. The goal is to find an amplitude where the minimum area is an acceptable value and the magnitude of the barrier function is approximately zero.

The barrier function is simply added to the original functional given in Equation (1). The modified CN functional for vertex $p$ now has the form

$$
F\left(\mathbf{x}_{p}\right)=\sum_{c \in \mathcal{C}(p)}\left(W^{c p} \frac{M^{c p}}{J^{c p}}+W^{c p-} \frac{M^{c p-}}{J^{c p-}}+W^{c p+} \frac{M^{c p+}}{J^{c p+}}+B^{c}\right)
$$

where $B^{c}$ is the barrier function for cell $c$. If $\Omega_{T}$ is the target minimum area, then the barrier function takes the form

$$
B^{c}=A_{B} \exp \left(-\frac{P_{B}}{\Delta \Omega_{B}}\left(\Omega_{T}-\Delta \Omega_{T}-\Omega_{c}\right)\right)
$$

where $A_{B}$ is the amplitude of the barrier function, $\Delta \Omega_{B}$ controls the width of the barrier function, $P_{B}$ controls the strength of the barrier function at the edges specified by $\Delta \Omega_{B}$, and $\Delta \Omega_{T}$ specifies how far the 
barrier function should be from the target area. In this work, $A_{B}$ is set to $10, P_{B}$ is set to $0.03, \Delta \Omega_{B}$ is set to $0.02 \Omega_{R}$, and $\Delta \Omega_{T}$ is set to $0.05 \Omega_{R}$, where the variable $\Omega_{R}$ is the reference area, as defined in Equation (11). Once the barrier has been added to the $\mathrm{CN}$ functional, the remainder of the mesh relaxation procedure is unchanged.

It is worth noting that for the quadrilateral cell geometry used in this work, the area of cell $c$ can be computed as

$$
\Omega_{c}=\frac{1}{2}\left(J^{c p-}+J^{c p+}\right) .
$$

This also allows the derivatives of the cell area, which are needed for the minimization procedure, to be easily evaluated since the derivatives of $J^{c p-}$ and $J^{c p+}$ were already computed for the original functional.

The weight amplitude adjustment works by modifying the weight function amplitude whenever the minimum cell area of the mesh is outside a specified bound. If $\Omega_{m}$ is the current minimum cell area for the mesh, then the weight amplitude is decreased whenever $\Omega_{m}<\Omega_{T}$. The amplitude is increased whenever $\Omega_{m}>\Omega_{T}+\delta \Omega_{T}$, where $\delta \Omega_{T}$ is the amount of leeway allowed for the final minimum area. In this work, $\delta \Omega_{T}$ is set to $0.02 \Omega_{R}$. The amplitude is adjusted at the beginning of every relaxation iteration. If $A^{i}$ is the current amplitude and $A^{i+1}$ is the updated amplitude, $A^{i+1}$ is expressed as

$$
A^{i+1}= \begin{cases}\max \left(A^{i} \sqrt{R_{T}}, 0.99 A^{i}\right) & \text { if } \Omega_{m}<\Omega_{T} \\ \min \left(A^{i} \sqrt{R_{\delta T}}, A^{i}+0.25\right) & \text { if } \Omega_{m}>\Omega_{T}+\delta \Omega_{T} \\ A^{i} & \text { otherwise }\end{cases}
$$

where

$$
R_{T}=\frac{\Omega_{m}}{\Omega_{T}} \quad \text { and } \quad R_{\delta T}=\frac{\Omega_{m}}{\Omega_{T}+\delta \Omega_{T}} .
$$

\subsection{Summary of method}

The new mesh relaxation method presented here can be summarized as the following process.

1. Generate an SDF or LOSDF from the given interface. If a level set method is used to track the interface, its corresponding re-distancing/re-initialization method can be used to generate an SDF. If the interface is given as an index function, an LOSDF can be created using the method from Section 3.3.

2. Compute the new barrier function amplitude from Equation (21).

3. Compute the weight function from the SDF or LOSDF using Equation (13) and the WENO reconstruction from Section 2.2.

4. Form the CN functional at each vertex using Equation (18) and then minimize it to compute a new position for all the vertices.

5. Update all the vertices with their new positions.

6. Return to step 1 and repeat until the desired mesh is obtained. 
Steps 1 and 4 are the most computationally expensive portions of the new algorithm. Although done in serial in the present manuscript, our preliminary estimates show these are easily GPU parallelizable, which will be explored in the future using node-level parallel programming models and multi-threading. In Nourgaliev et al. [17], we explored GPU parallelization of a high-order DG re-distancing algorithm and found significant speedup and excellent scalability when utilizing GPUs through the RAJA portability layer [20]. The parallelization techniques from that work can be directly applied to the new mesh relaxation algorithm.

\section{Static Interface Examples}

The first set of example meshes will be for static interfaces. In all the examples, we begin with a uniform orthogonal mesh and apply the mesh relaxation method for a fixed number of iterations. These static interface examples can be viewed as a mesh generation procedure for purely Eulerian simulations or as the initial mesh generation for a dynamic interface simulation. All of the examples will utilize an index function to prescribe the interface location since this is the more general approach. Nearly identical results can be obtained by using a level set method to track the interface's location, which will be demonstrated in the first example.

\subsection{Sinusoidal}

The first example will be for a sinusoidal interface, which was selected to represent the linear growth stage of the Rayleigh-Taylor instability. The index function used to define the interface is shown in Figure 4a on the initial $41 \times 40$ mesh. The LOSDF generated from this index function is depicted in Figure $4 \mathrm{~b}$. The exact SDF is also included in the figure for comparison. The LOSDF does a very good job of approximating the exact SDF, especially considering it was generated from an index function and the overall simplicity of the algorithm. For this geometry, we wanted to refine symmetrically about the interface with a target area of $0.4 \Omega_{R}$. This can be achieved with the weight function shown in Figure 5 , which was generated using the parameters given in Table 1.

To ensure the final converged mesh is independent of the initial weight amplitudes $\left(A^{0}\right)$, three different values were used: 2,5 , and 10. A history of the convergence metric and weight amplitude for all three cases is shown in Figure 6. Although the initial transient portions of the profiles are different, all the cases essentially converged by approximately 300 iterations. A comparison of the mesh after 300 iterations for the $A^{0}=2$ and $A^{0}=10$ cases is shown in Figure 7a. The meshes use periodic boundary conditions in the $x$ direction, which is the source of the distortion along the right and left edges of the meshes. As expected, after 300 iterations the two meshes are nearly identical. Although not shown, the $A^{0}=5$ case compares equally well. The meshes possess the desired symmetric refinement about the interface and had no difficulty following the geometry. Figure $7 \mathrm{~b}$ provides a comparison of the mesh generated from the index function 


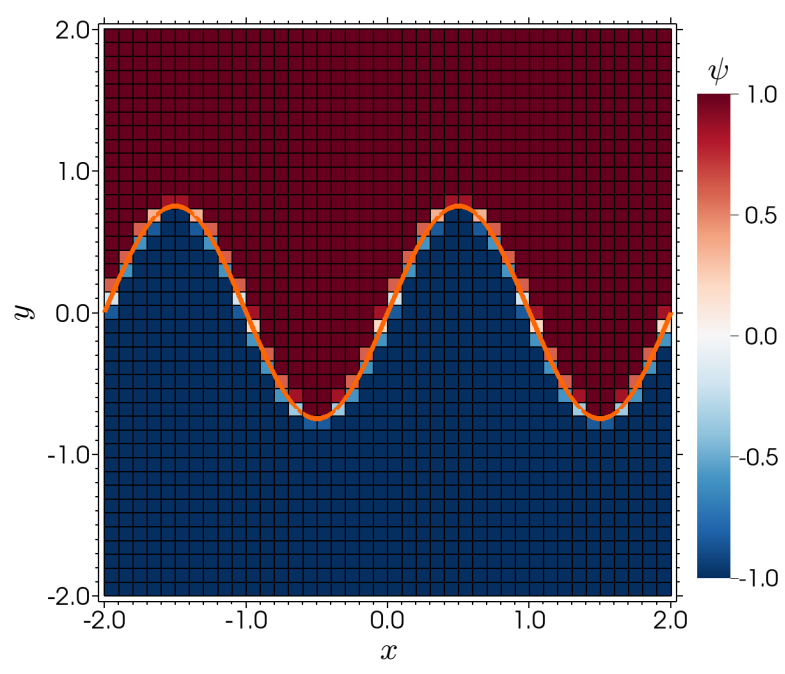

(a) Index function. Interface shown as -

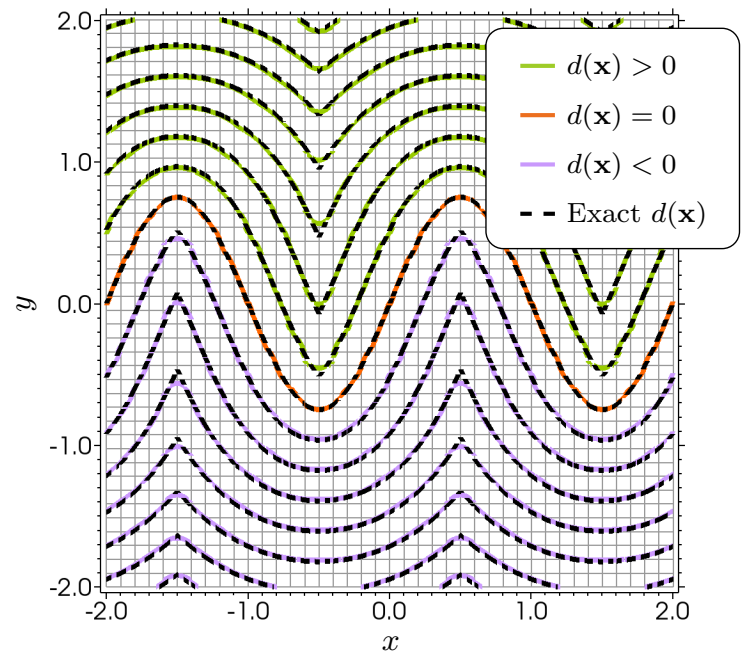

(b) LOSDF. 15 evenly-spaced levels from -1.5 to 1.5 .

Figure 4: Index function and LOSDF for the static sinusoidal interface on initial uniform mesh.

\begin{tabular}{cccc}
\hline$d_{o}^{+}$ & $d_{i}^{+}$ & $d_{i}^{-}$ & $d_{o}^{-}$ \\
\hline 0.6 & 0.1 & -0.1 & -0.6 \\
\hline
\end{tabular}

Table 1: Weight function parameters for the static sinusoidal interface.

and from the exact SDF. Although slight differences are present, the two meshes are very similar with neither being clearly superior. This is expected given how closely the LOSDF approximates the exact SDF (Figure 4b).

A history of the minimum and maximum cell area is presented in Figure 8 for the three different cases. These plots once again show that the meshes have converged after 300 iterations. During the initial transients, the minimum cell area for each mesh quickly decreased till it reached the target value, after which it did not diverge from that value. This is the type of behavior the barrier function and weight adjustment procedure were meant to produce. Table 2 provides the weight amplitude and areas for the three cases after 1000 iterations. All three initial amplitudes converged to the same weight amplitude, minimum area, and maximum area. The minimum area for all three cases were very close to the specified target value of $0.4 \Omega_{R}$.

\subsection{Cruciform}

The second static interface example is the cruciform geometry prescribed by the index function shown in Figure 9a. A $60 \times 60$ mesh is used for this example. The length of each arm of the cruciform is 1 and the thickness is 0.2 . The corners of the cruciform are rounded with a radius of 0.1 . The cruciform is rotated $30^{\circ}$ from the horizontal. This geometry was selected to test the mesh relaxation method on a complex geometry 


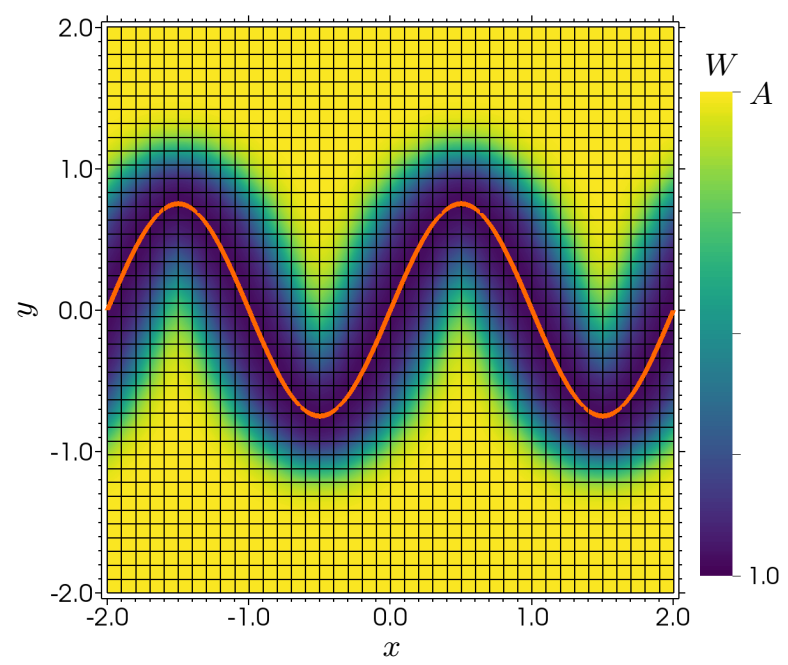

Figure 5: Weight function for the static sinusoidal interface on initial uniform mesh. Interface shown as —.

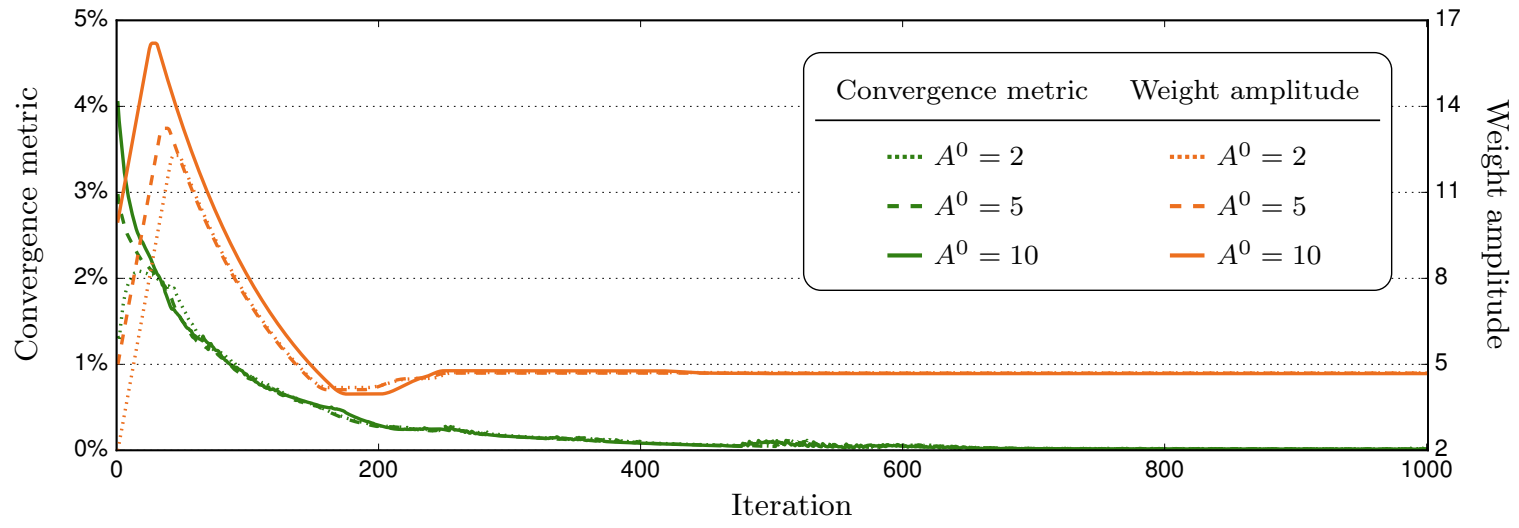

Figure 6: Convergence metric and weight amplitude for the static sinusoidal interface.

\begin{tabular}{cccc}
\hline Initial weight amplitude & Weight amplitude & Minimum area & Maximum area \\
\hline 2 & 4.70 & 0.404 & 1.72 \\
5 & 4.69 & 0.404 & 1.72 \\
10 & 4.68 & 0.405 & 1.72 \\
\hline
\end{tabular}

Table 2: Weight function amplitude and cell area values for the static sinusoidal interface after 1000 iterations. Cell area values are non-dimensionalized by reference area. 


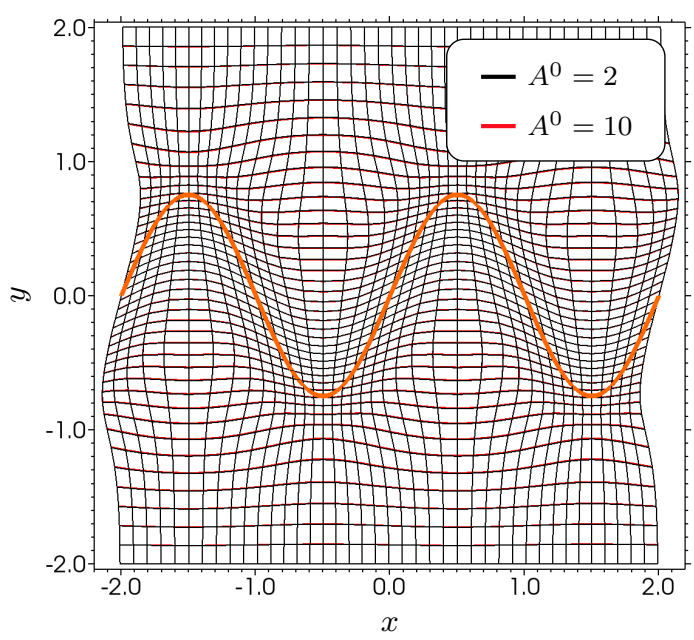

(a) Comparison of $A^{0}=2$ and $A^{0}=10$ meshes.

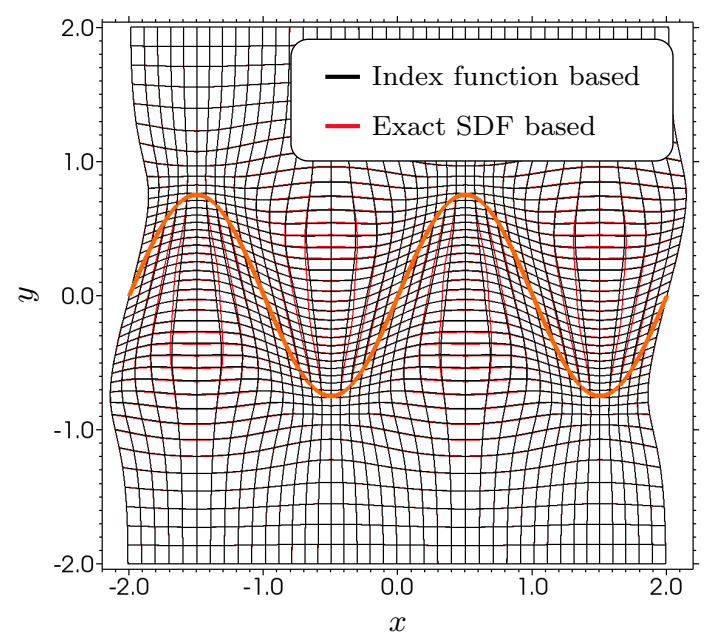

(b) Comparison of meshes generated from the index function and the exact SDF.

Figure 7: Mesh after 300 iterations for the static sinusoidal interface. Interface shown as —.

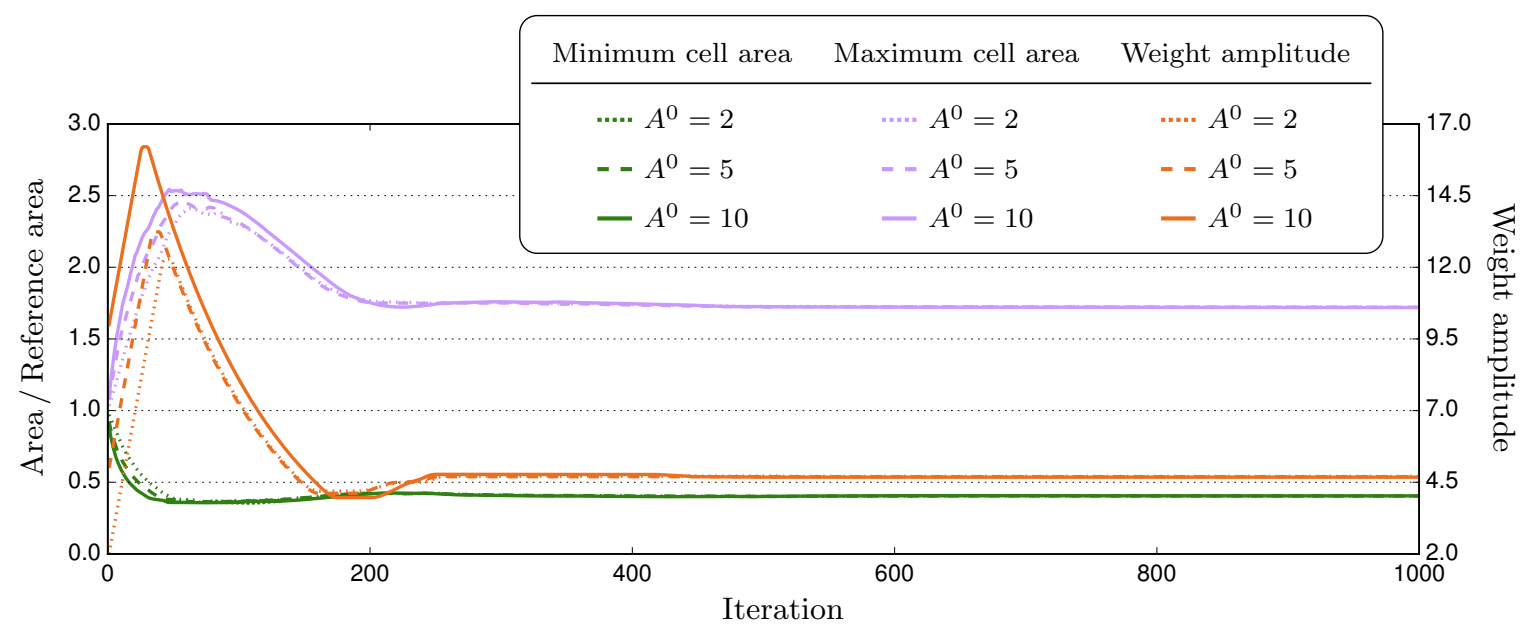

Figure 8: Minimum cell area, maximum cell area, and weight amplitude for the static sinusoidal interface. 


\begin{tabular}{cccc}
\hline$d_{o}^{+}$ & $d_{i}^{+}$ & $d_{i}^{-}$ & $d_{o}^{-}$ \\
\hline 0.6 & 0.1 & -1.0 & -2.0 \\
\hline
\end{tabular}

Table 3: Weight function parameters for the static cruciform interface.

with thin regions requiring increased resolution. In addition, this geometry has a number of discontinuities in the slope of the exact SDF. Figure 9b shows the LOSDF and the exact SDF for this geometry. For the positive values, the discontinuities are located between the arms of the cruciform and appear to originate at the corners. Although not evident with the current contours, there are also discontinuities in the negative values along the centerline of each arm. For this case, we wanted to generate a high resolution region with approximately uniform cells inside the cruciform and then transition to a coarser resolution away from the interface. This can be generated using the weight function depicted in Figure 10. The parameters for this weight function are given in Table 3 .

Just as in the sinusoidal interface, three different initial weight amplitudes are used here: 2,15 , and 30. The convergence metric and weight amplitude histories for all three cases are presented in Figure 11. The plots show that only the weight amplitude for the $A^{0}=30$ case has reached a steady value after 2000 iterations (the amplitude actually converged after approximately 1000 iterations). The results suggest that higher initial values may provide faster convergence of the weight amplitude, which in turn should allow the mesh to converge faster. A comparison of the mesh after 2000 iterations for the three different initial amplitudes is provided in Figure 12, with the $A^{0}=30$ case used as a reference. As expected from the convergence metric results, there are differences between the meshes. However, all three meshes conformed to the geometry and had no difficulties from the discontinuities in the SDF. Although the weight amplitude for two of the meshes have not reached a final value, within the cruciform, the meshes are nearly identical and provided the desired resolution. Initially, the arms of the cruciform are highly under-resolved, with only about two cells covering the width of the arms. After 2000 iterations, the resolution has doubled to approximately four cells.

Figure 13 shows the history of the minimum and maximum cell area for all three cases and the values after 2000 iterations are provided in Table 4. Despite the differences in the weight function amplitude and the maximum cell area, the minimum cell area for each case follows a very similar trend. The value quickly decreases to the target value and then remains roughly constant for the remainder of the simulation. This is the reason the meshes within the cruciform for all three cases are similar. This also means that meshes need not be relaxed to a final weight amplitude and tight convergence tolerance to obtain a desired minimum area. 


\begin{tabular}{cccc}
\hline Initial weight amplitude & Weight amplitude & Minimum area & Maximum area \\
\hline 2 & 8.29 & 0.273 & 1.91 \\
15 & 7.42 & 0.259 & 2.03 \\
30 & 6.75 & 0.262 & 1.96 \\
\hline
\end{tabular}

Table 4: Weight and cell area values for the static cruciform interface after 2000 iterations. Cell area values are nondimensionalized by reference area.

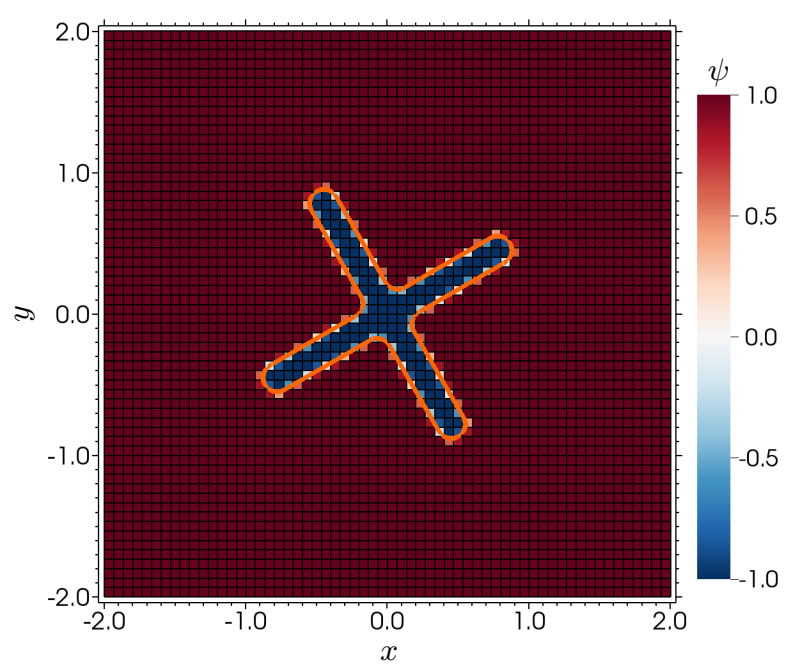

(a) Index function. Interface shown as -.

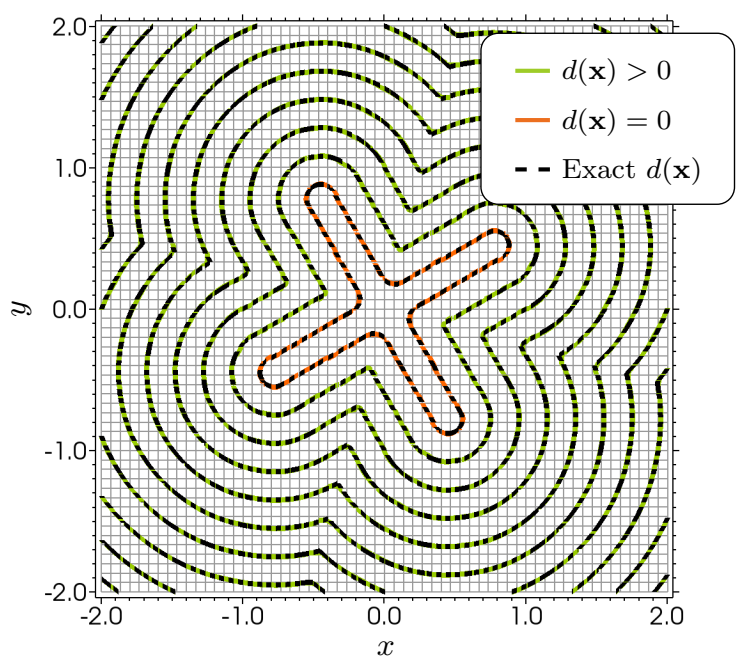

(b) LOSDF. 10 evenly-spaced levels from 0.0 to 1.8 .

Figure 9: Index function and LOSDF for the static cruciform interface on initial uniform mesh.

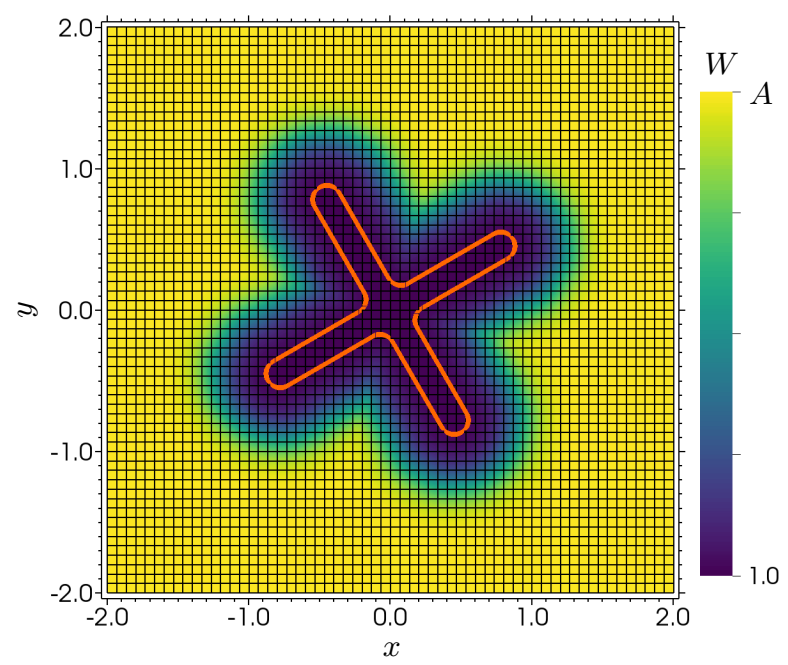

Figure 10: Weight function for the static cruciform interface on initial uniform mesh. Interface shown as - 


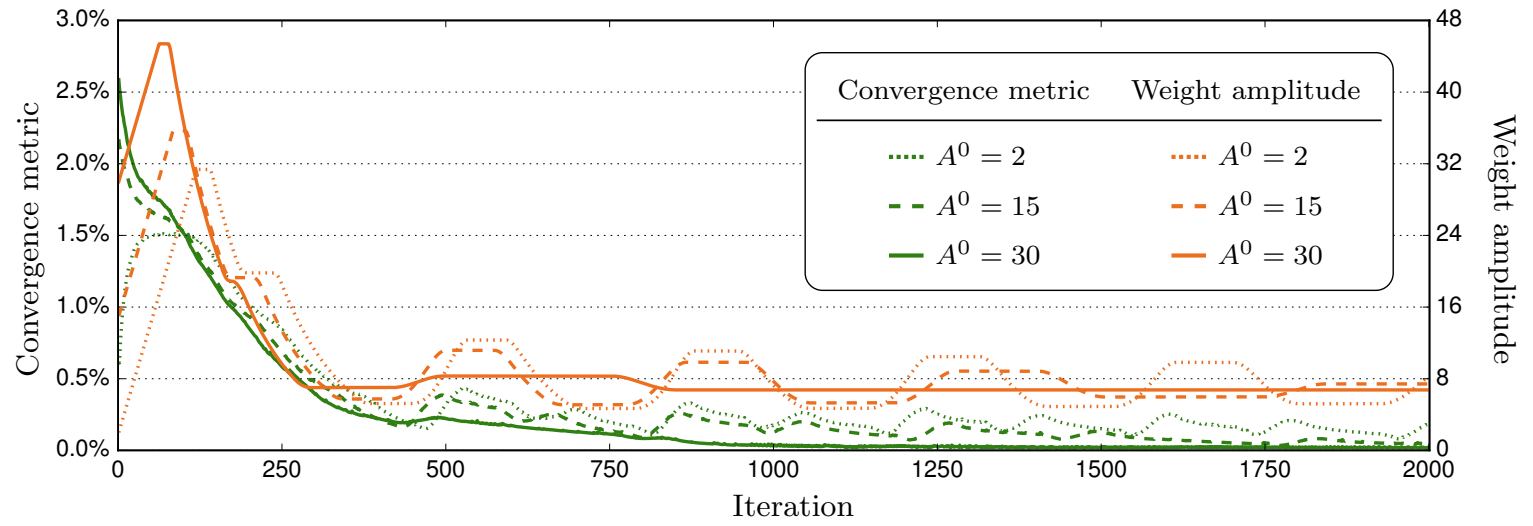

Figure 11: Convergence metric and weight amplitude for the static cruciform interface.

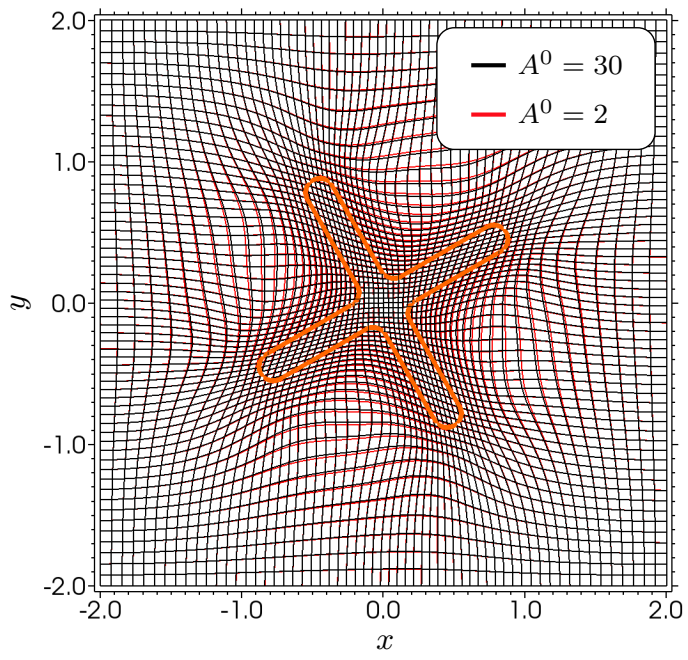

(a) Comparison of $A^{0}=30$ and $A^{0}=2$ meshes.

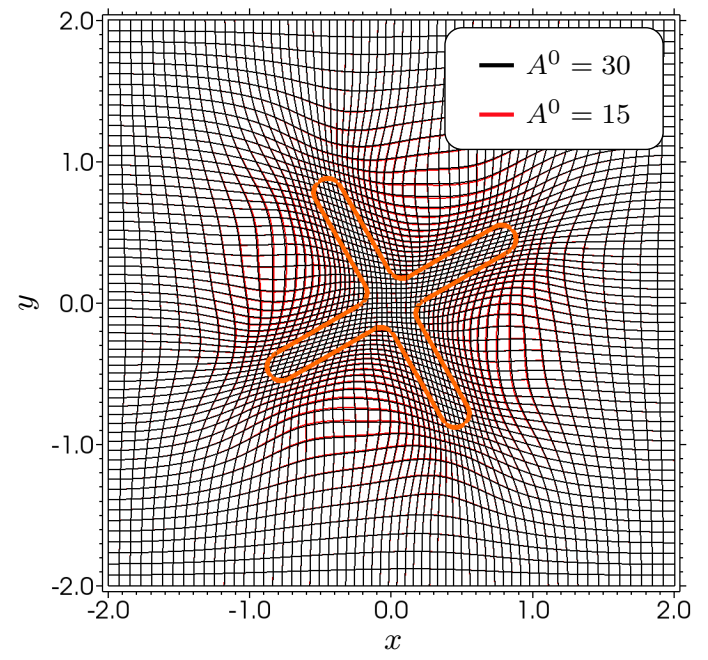

(b) Comparison of $A^{0}=30$ and $A^{0}=15$ meshes.

Figure 12: Meshes after 2000 iterations for the static cruciform interface. Interface shown as —. 


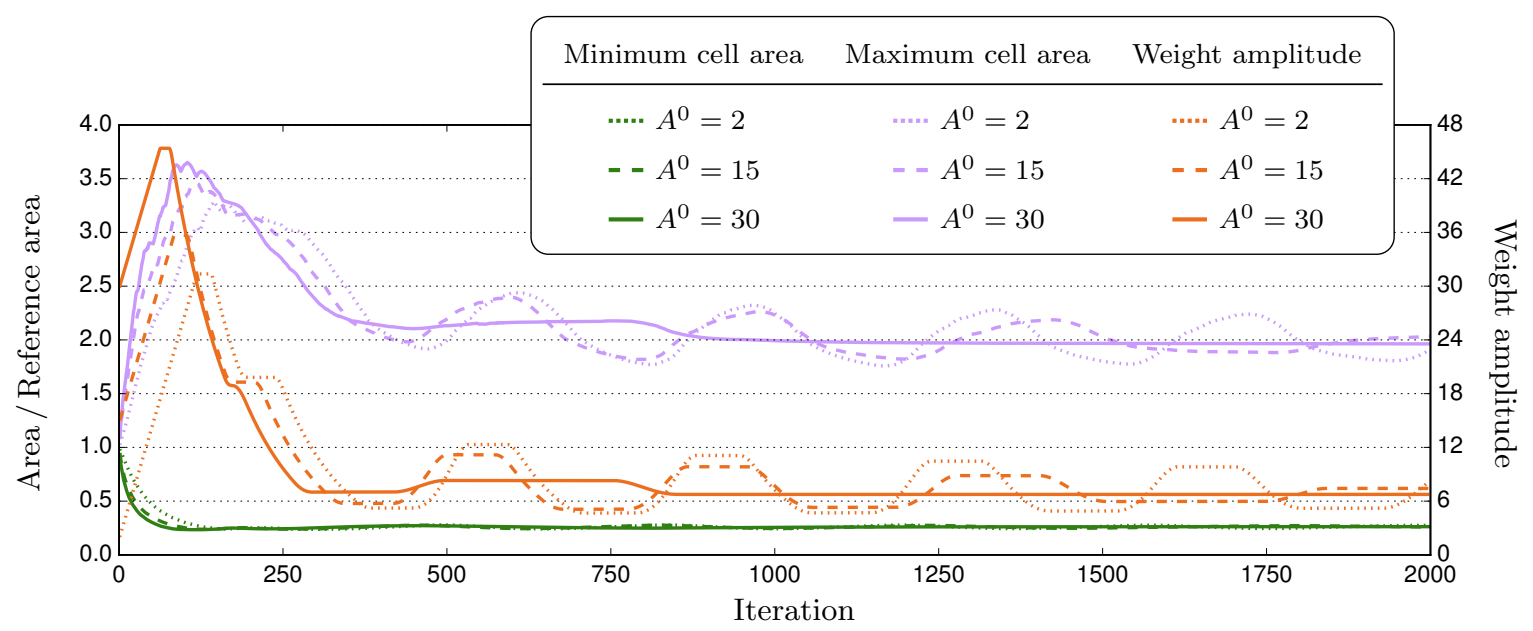

Figure 13: Minimum cell area, maximum cell area, and weight amplitude for the static cruciform interface.

\subsection{Three circles}

In multi-material simulations, different material interfaces are usually tracked using separate level sets or index functions. The next static interface example will mimic this set up by generating a mesh for three circular interfaces where each circle is defined by a separate index function. This would be analogous to a simulation with four different materials. Figure 14a shows the three circular interfaces and the index function used to specify the top circle. The LOSDF for that index function is shown in Figure 14b. A $60 \times 60$ mesh is used for this example. Since each circle has its own index function and LOSDF, separate weight functions with different parameters can be generated for each interface. This is very useful for multimaterial simulations since the different interfaces may vary greatly in size and shape, which may result in them requiring different levels of refinement. It should be noted, if different amplitudes are used for the weight functions, they should be scaled such that they have the same maximum (but different minimums). Although the circles in this work are all roughly the same size, different parameters will be used for each circle to demonstrate the flexibility of the weight function framework provided in this paper.

The weight function used for each circle is shown in Figure 15. For the top circle, the weight function parameters were selected to provide a uniform resolution within the circle and gradually reduce the refinement away from the interface. The weight function for the lower right circle was set to provide increased resolution along the outer edge of the circle. A similar approach was used for the lower left, except the clustering is applied along the inner edge of the circle. The weight function and geometric parameters for each circle are provided in Table 5. Although separate weight functions can be generated for each interface, only a single weight function can be used during the mesh relaxation. The different functions can be easily combined by simply taking the minimum of the three functions when creating the $\operatorname{rDG}\left(\mathrm{P}_{1} \mathrm{P}_{2}\right)$ reconstruction of the combined weight function. The combined weight function is shown in Figure 15d. When multiple level 


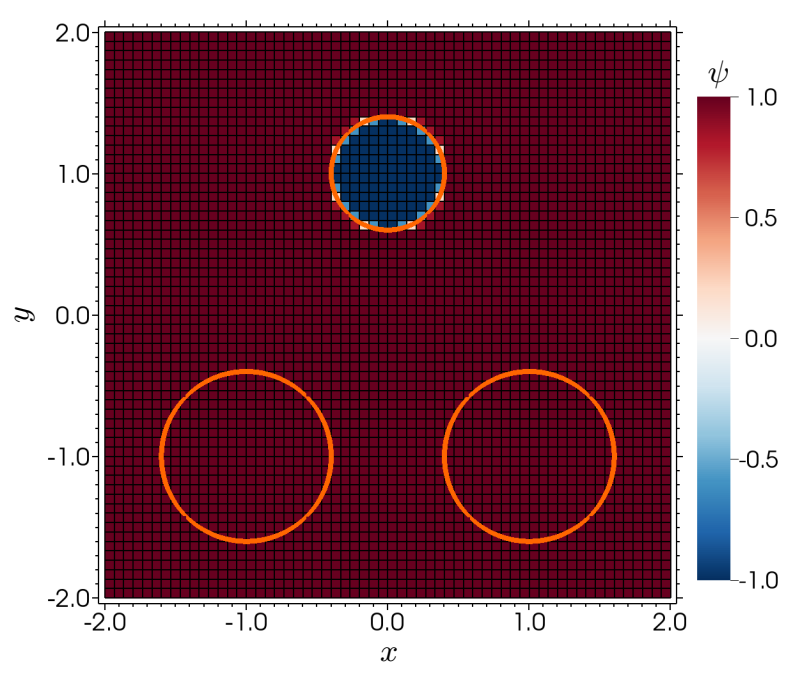

(a) Index function. Interfaces shown as - .

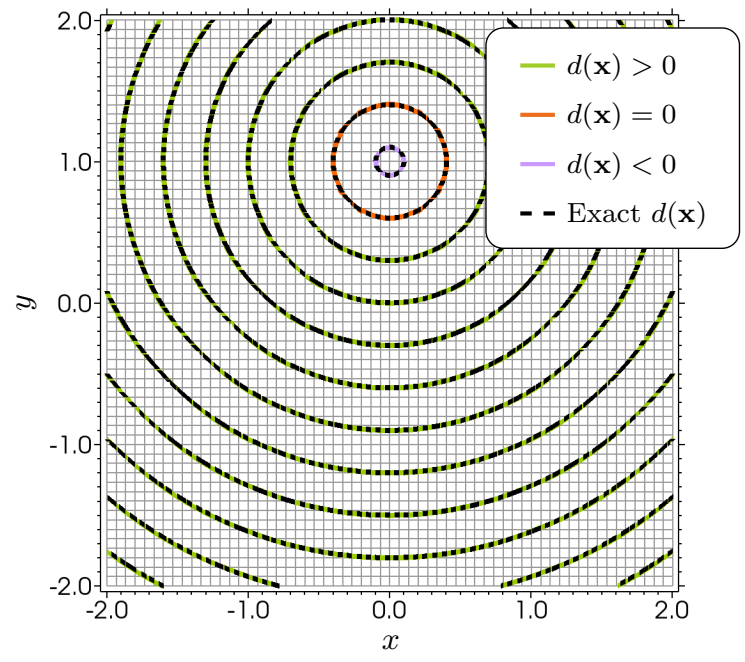

(b) LOSDF. 12 evenly-spaced levels from -0.3 to 3.0.

Figure 14: Index function and LOSDF for the top circle of the three circles example on initial uniform mesh.

\begin{tabular}{ccccccc}
\hline Circle & Center & Radius & $d_{o}^{+}$ & $d_{i}^{+}$ & $d_{i}^{-}$ & $d_{o}^{-}$ \\
\hline Top & $(0,1)$ & 0.4 & 0.6 & 0.0 & -4.0 & -5.0 \\
Lower right & $(1,-1)$ & 0.6 & 0.6 & 0.1 & 0.0 & -0.4 \\
Lower left & $(-1,-1)$ & 0.6 & 0.4 & 0.0 & -0.1 & -0.6 \\
\hline
\end{tabular}

Table 5: Geometric and weight function parameters for the three circles example.

sets are used, the interdependence of the weight function amplitude for each level set should be specified. One option is to specify an initial value for each level set and then maintain the ratio between the different amplitudes. Another option is to specify a minimum cell area for each interface and allow the amplitudes to vary independently. The optimal approach is likely to be problem specific. For this example, we use the same weight function amplitude for each interface during the relaxation process. However, the value is allowed to adjust in order to obtain a minimum area of $0.35 \Omega_{R}$.

The mesh after 2000 relaxation iterations is shown in Figure 16. The mesh is refined in the exact locations specified by the weight functions with a minimum cell area of $0.349 \Omega_{R}$. The top circle has a uniform resolution throughout the interior, while the lower right and left circles are refined along their outer and inner edges, respectively. A history of the convergence metric and weight function amplitude is shown in Figure 17 and a history of the minimum and maximum cell areas is provided in Figure 18. The plots clearly show that the weight amplitude and the maximum cell area are still fluctuating. However, just as in the previous examples, the requested minimum cell area is obtained very early in the relaxation process. As a result, the method could have been stopped much earlier if the mesh was deemed acceptable. 


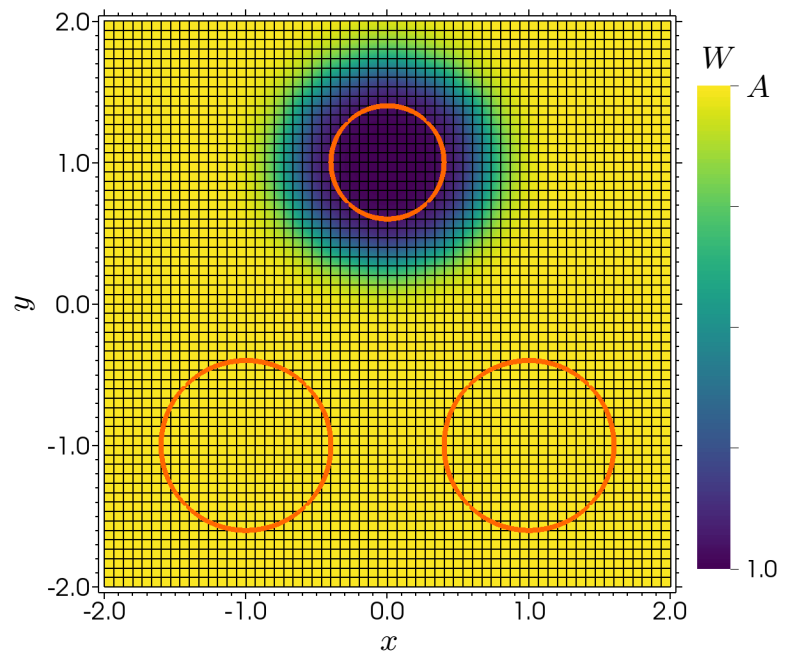

(a) Weight function for top circle.

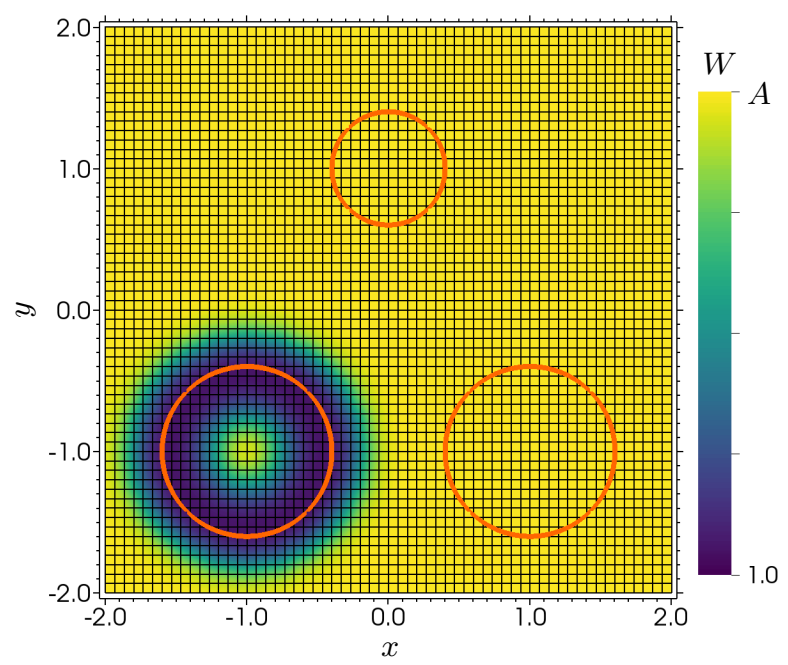

(c) Weight function for lower left circle.

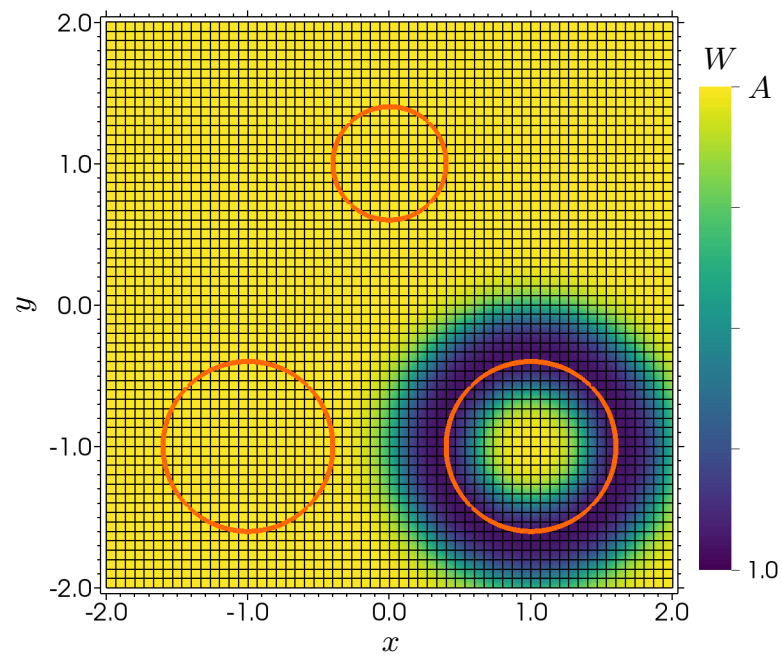

(b) Weight function for lower right circle

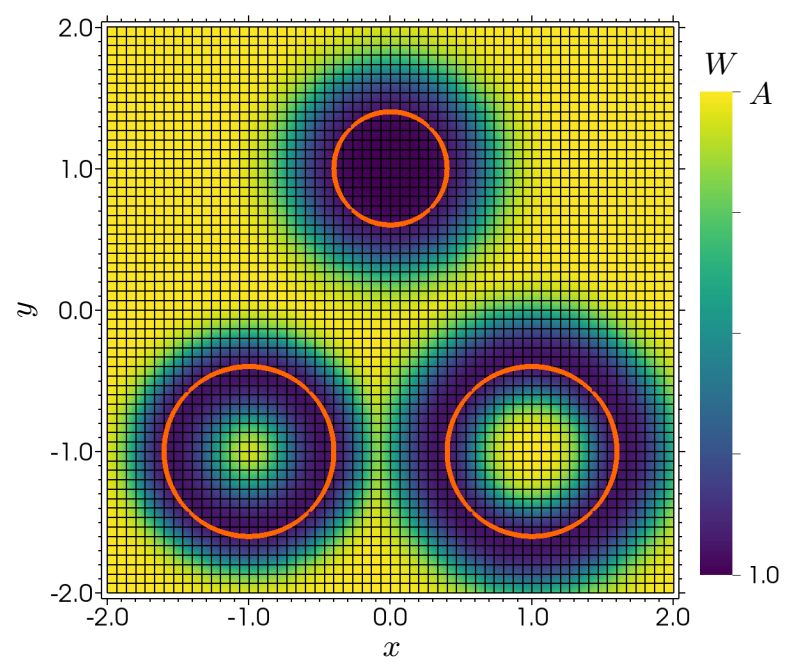

(d) Combined weight function.

Figure 15: Weight functions for the three circles example on initial uniform mesh. Interfaces shown as - . 


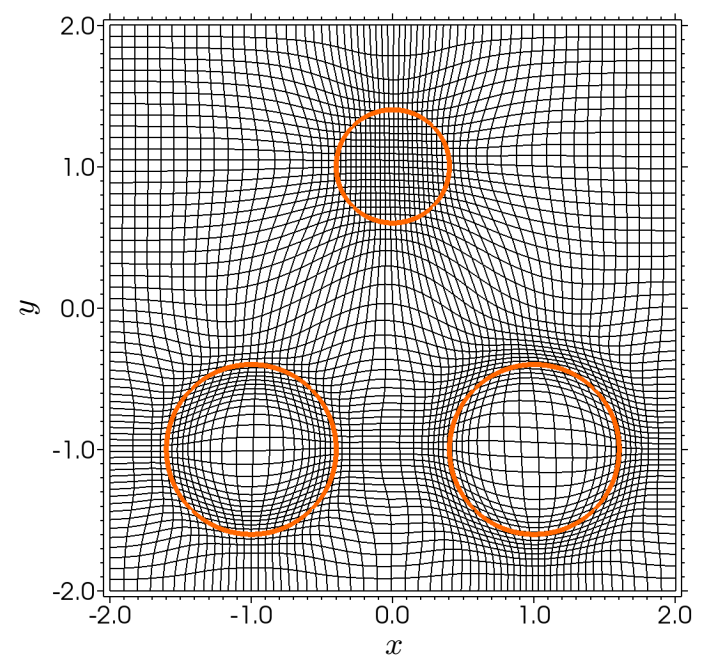

Figure 16: Mesh after 2000 iterations for the three circles example. Interfaces shown as —.

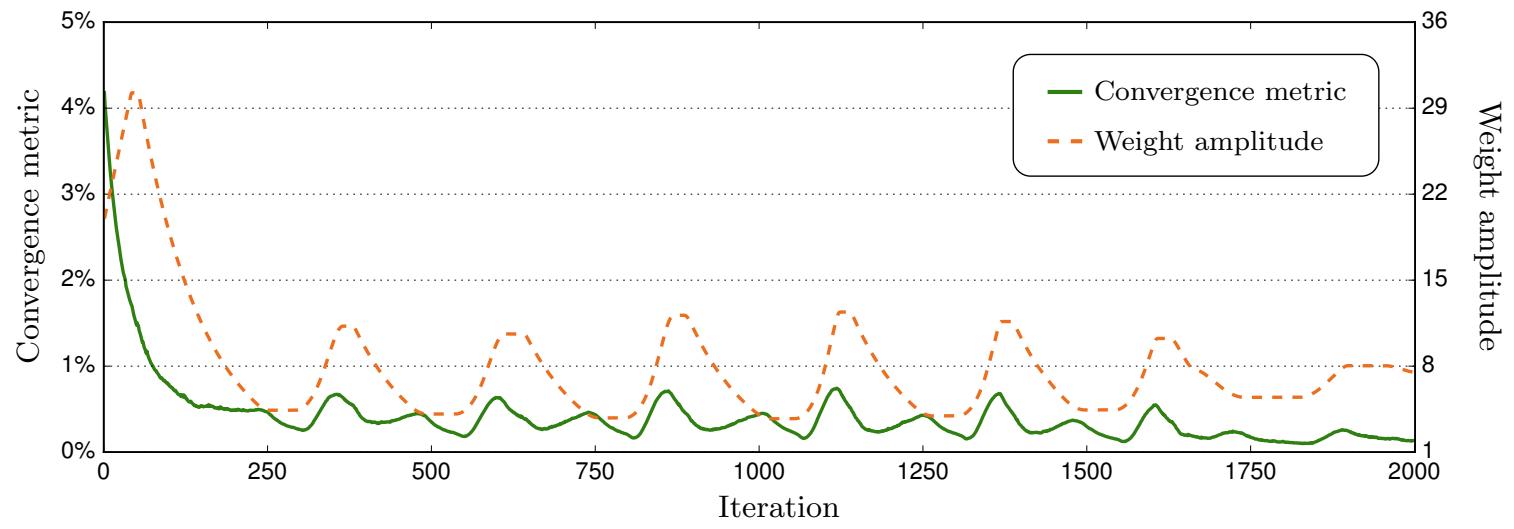

Figure 17: Convergence metric and weight amplitude for the three circles example.

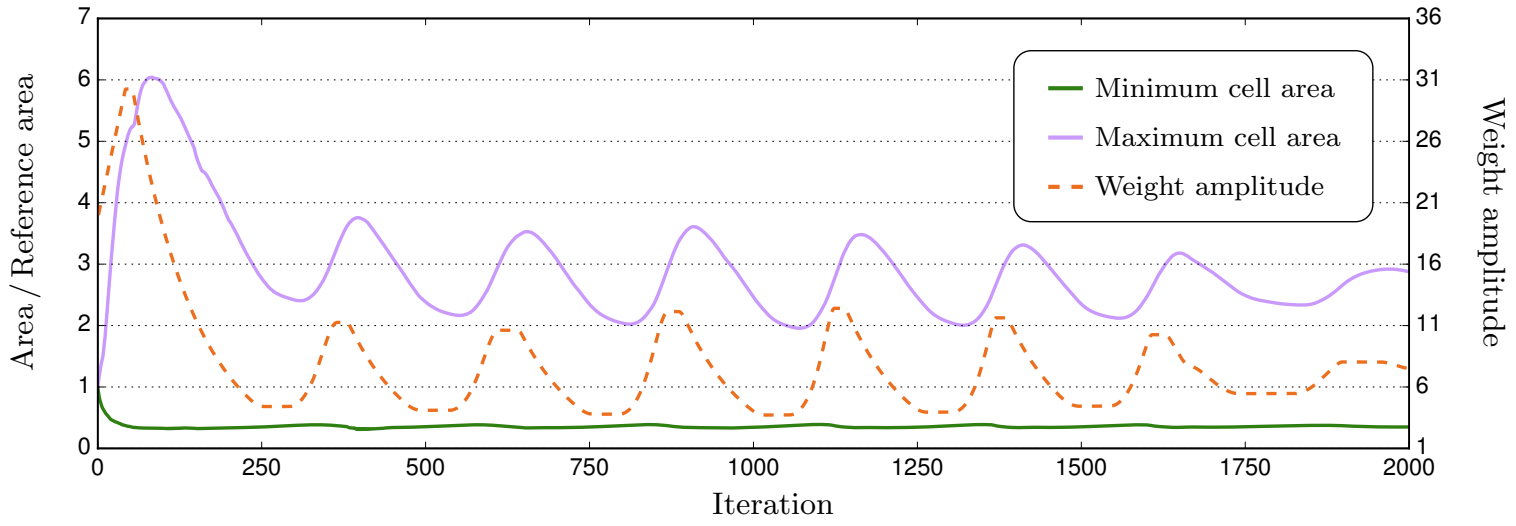

Figure 18: Minimum cell area, maximum cell area, and weight amplitude for the three circles example. 


\begin{tabular}{cc}
\hline Material Number & Level set value \\
\hline 1 & $\phi_{1}(\mathbf{x})<0$ \\
2 & $\phi_{2}(\mathbf{x})<0$ \\
$\vdots$ & $\vdots$ \\
$M-1$ & $\phi_{M-1}(\mathbf{x})<0$ \\
$M$ & $\phi_{1}(\mathbf{x})>0, \phi_{2}(\mathbf{x})>0, \ldots, \phi_{M-1}(\mathbf{x})>0$ \\
\hline
\end{tabular}

Table 6: Level set values for identifying individual materials in an $M$-material problem.

\subsection{T-junction}

The final static example is for a T-junction of three different materials. In general, the location of $M$ different materials can be specified by $M-1$ level set functions. Each $m$ th level set separates the $m$ th material from all the others. In this work, we assume the material is located in the negative portion of each level set. The final $M$ th material is defined by the superposition of the positive zones for all the level sets, that is, anywhere none of the other materials are present. This is summarized in Table 6 , where $\phi_{m}(\mathbf{x})$ is the level set for material $m$. Since the LOSDF is a type of level set, $\phi_{m}(\mathbf{x})$ can be replaced with $d_{m}(\mathbf{x})$, which is the LOSDF for material $m$. Therefore, the three material T-junction geometry used for this example can be specified with two index functions, which will result in two LOSDFs. The extent of the first and second materials are specified by the index functions shown in Figure 19. The functions are shown on the initial uniform $40 \times 40$ mesh. As the figure shows, the T-junction consist of three linear interfaces originating at the origin of the domain. With the positive $x$-axis as $0^{\circ}$, the three interfaces are at $-55^{\circ}, 50^{\circ}$, and $145^{\circ}$.

Figure 19 also shows the LOSDF for materials 1 and 2, which were generated from their corresponding index functions. Similar to the index function, the third material is located anywhere both LOSDFs are positive. Over most of the domain, the LOSDFs do a good job of approximating the analytic SDFs. The exception to this are the areas where the interface crosses the domain boundary, where some of the contours of the LOSDF slightly diverge from the exact SDF and move closer to the interface. This is due to the analytic solutions assuming that the interfaces continue pass the edges of the domain, while the LOSDFs assume the interfaces end at the domain boundary. This small discrepancy did not appear to degrade the final mesh.

Just as in the last example, a separate weight function is generated for each LOSDF and they are then merged by taking their minimum values. The individual and combined weight functions are shown in Figure 20. The parameters for the individual weight functions were selected to cluster cells along the inner/negative portion of the interfaces. The same values were used for both weight functions and are given in Table 7. In addition, the same weight function amplitude is used for both weight functions, but the value 


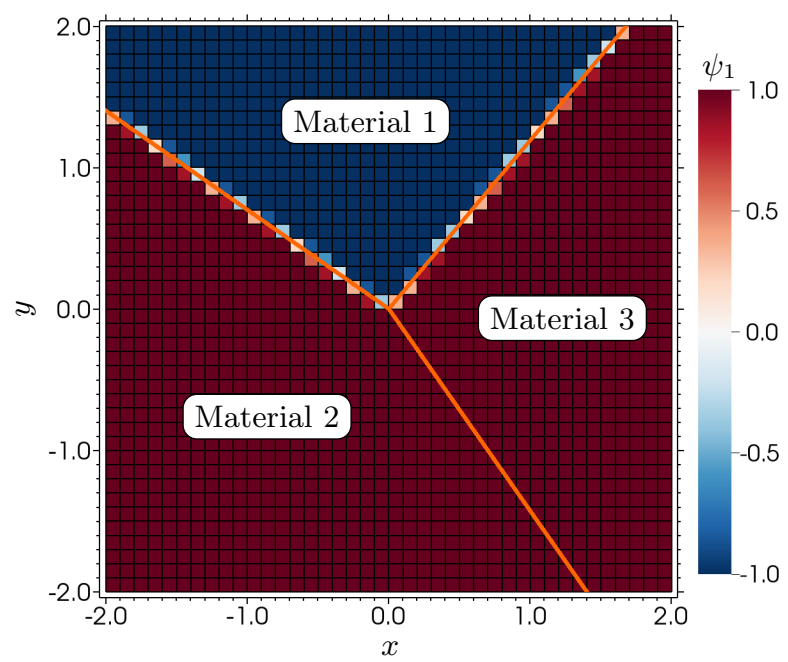

(a) Index function for material 1. Interfaces shown as - .

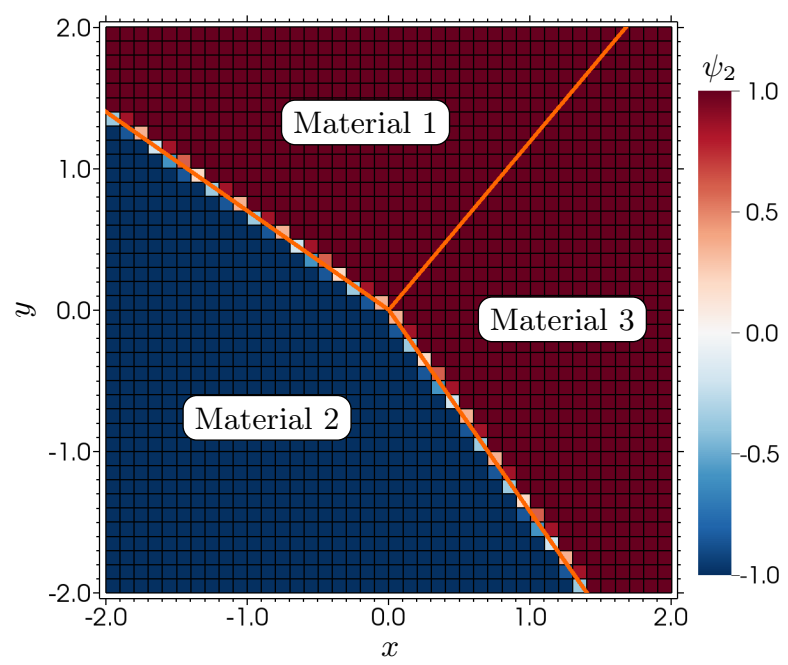

(c) Index function for material 2. Interfaces shown as -

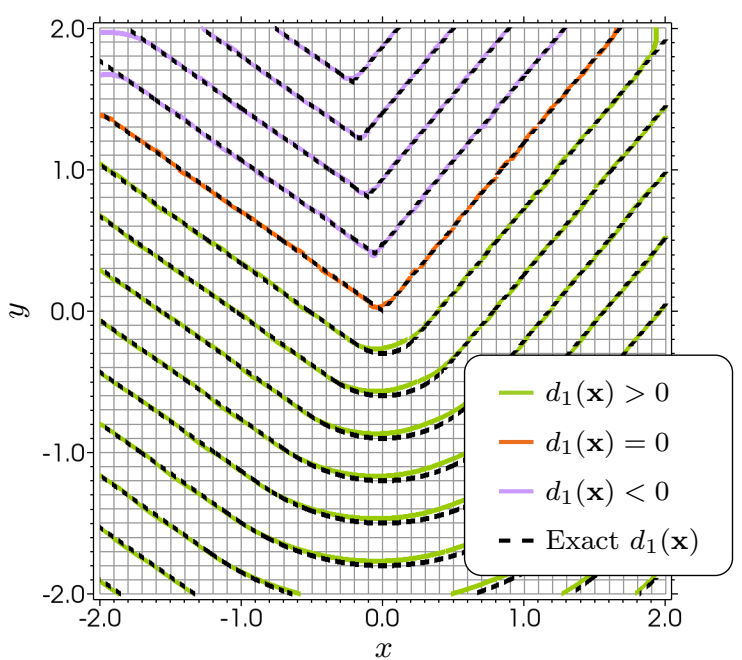

(b) LOSDF for material 1. 19 evenly-spaced levels from -2.7 to 2.7

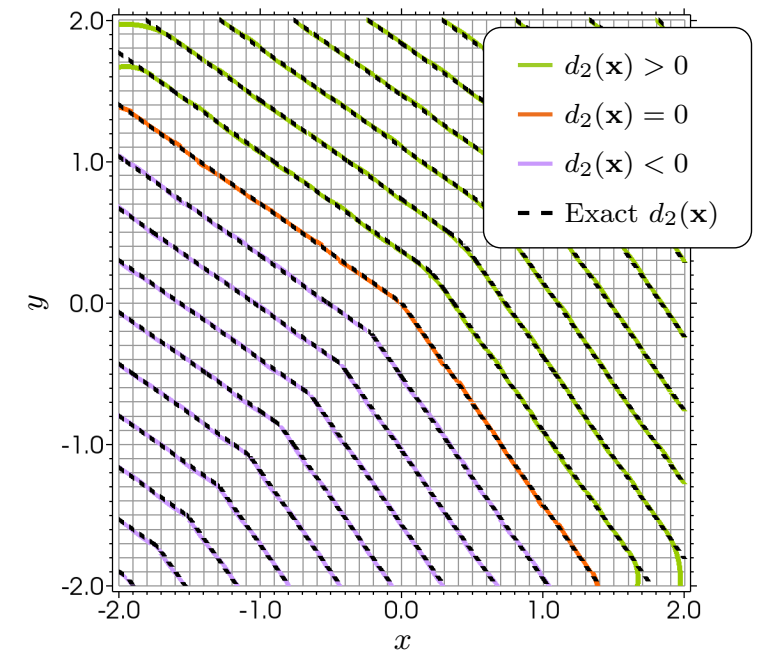

(d) LOSDF for material 2. 19 evenly-spaced levels from -2.7 to 2.7 .

Figure 19: Index functions and LOSDFs for the T-junction example on initial uniform mesh. 


\begin{tabular}{cccc}
\hline$d_{o}^{+}$ & $d_{i}^{+}$ & $d_{i}^{-}$ & $d_{o}^{-}$ \\
\hline 0.4 & 0.0 & -0.1 & -0.8 \\
\hline
\end{tabular}

Table 7: Weight function parameters for the T-junction example.

was allowed to change to obtain a target minimum cell area of $0.35 \Omega_{R}$. When the functions are combined, this creates a symmetric distribution along the interface between materials 1 and 2, while maintaining the one-sided refinement for the remaining interfaces.

The mesh generated after 1000 interactions is shown in Figure 21. The resulting mesh has been refined along the inner edge of materials 1 and 2, just as their individual weight functions specified. The relaxation method had no difficulties dealing with the triple point where the three materials meet. The convergence metric and weight amplitude histories are shown in Figure 22. Most of the initial transients have occurred in the first 400 iterations, after which the convergence metric has reached an acceptable value and the weight amplitude is no longer under going large fluctuations. Histories of the minimum and maximum cell areas are shown in Figure 23. The final minimum cell area of the mesh is $0.359 \Omega_{R}$.

\section{Dynamic Interface Examples}

The examples in the previous section involved using mesh relaxation to increase refinement near static interfaces. The eventual goal of the weighted $\mathrm{CN}$ relaxation method presented in this work is to be integrated into an ALE framework for use with dynamic interfaces. To demonstrate the potential of the method for ALE simulations, we will show two dynamic interface examples. For both examples, the interfaces will be prescribed by an index function.

The dynamic simulations will consist of two steps. First, the interfaces will be kept fixed and the mesh will be relaxed for a number of iterations starting from a uniform mesh. This will generate the initial mesh to be used during the second portion of the simulation. The dynamic examples in this section will start with the results from the static sinusoidal and static cruciform examples, as the initial mesh. In the second part, the interface will be moved with a prescribed motion and a constant CFL number. After each time step, the mesh relaxation method will be employed until a convergence tolerance of $0.25 \%$ is reached. A minimum of one relaxation step will always be used.

\subsection{Sinusoidal}

The first dynamic example is a "decaying and growing sinusoidal." The initial geometry of the interface and the parameters of the weight function are identical to the static sinusoidal used in Section 4.1. When the interface is set into motion, its amplitude will decay with a non-dimensional velocity of 1 . Since the initial 


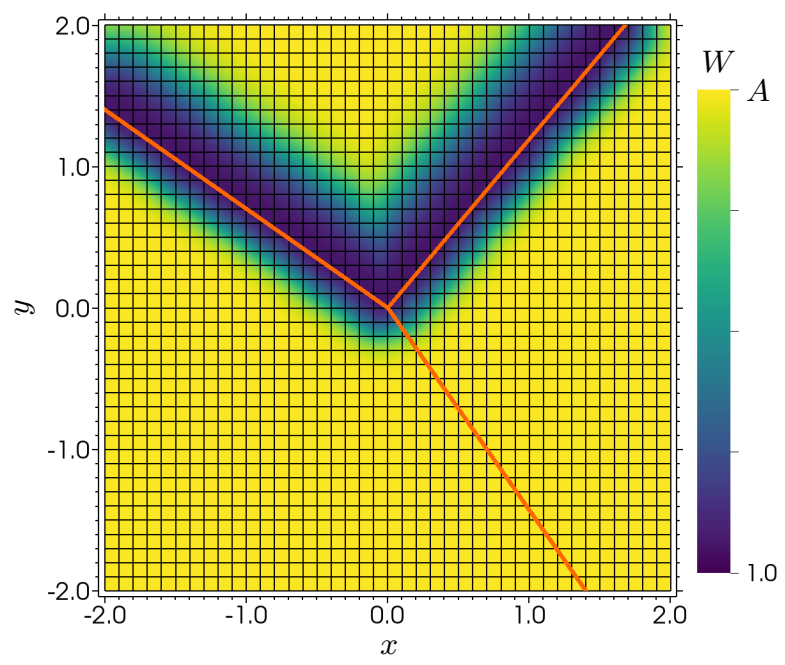

(a) Weight function for material 1 .

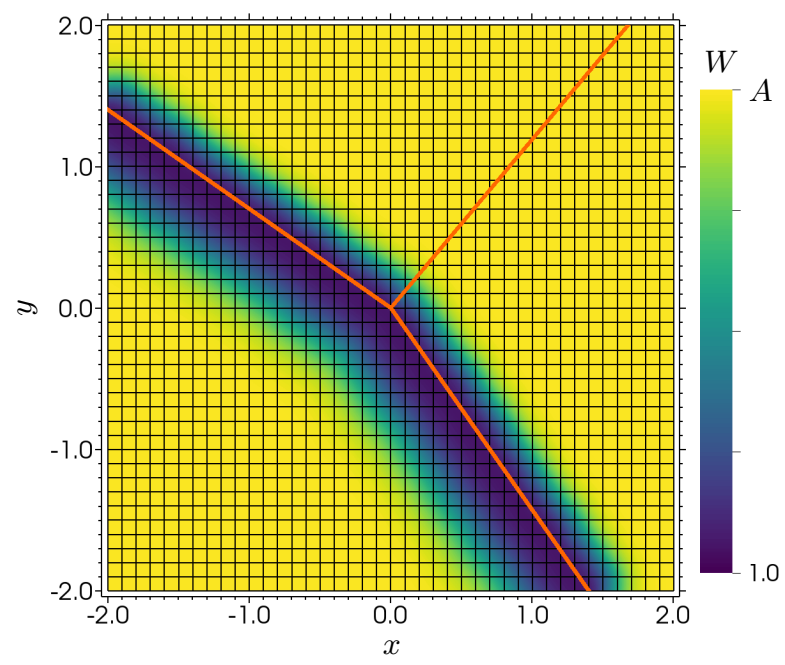

(b) Weight function for material 2.

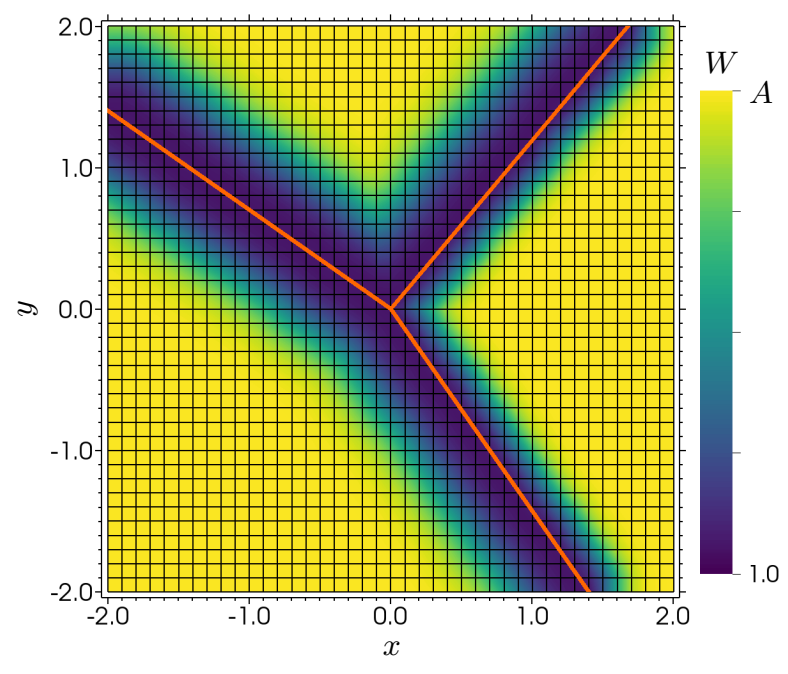

(c) Combined weight function.

Figure 20: Weight functions for the T-junction example on initial uniform mesh. Interfaces shown as - 


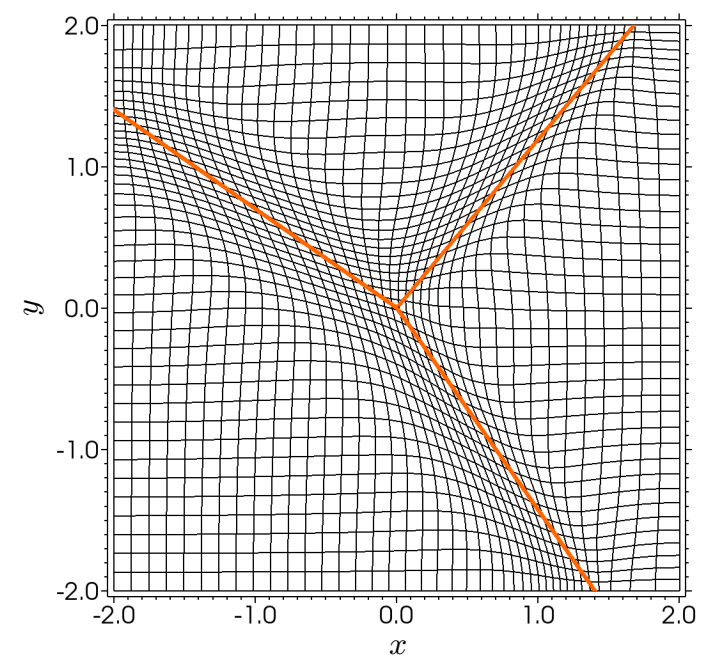

Figure 21: Mesh after 1000 iterations for the T-junction example. Interfaces shown as —.

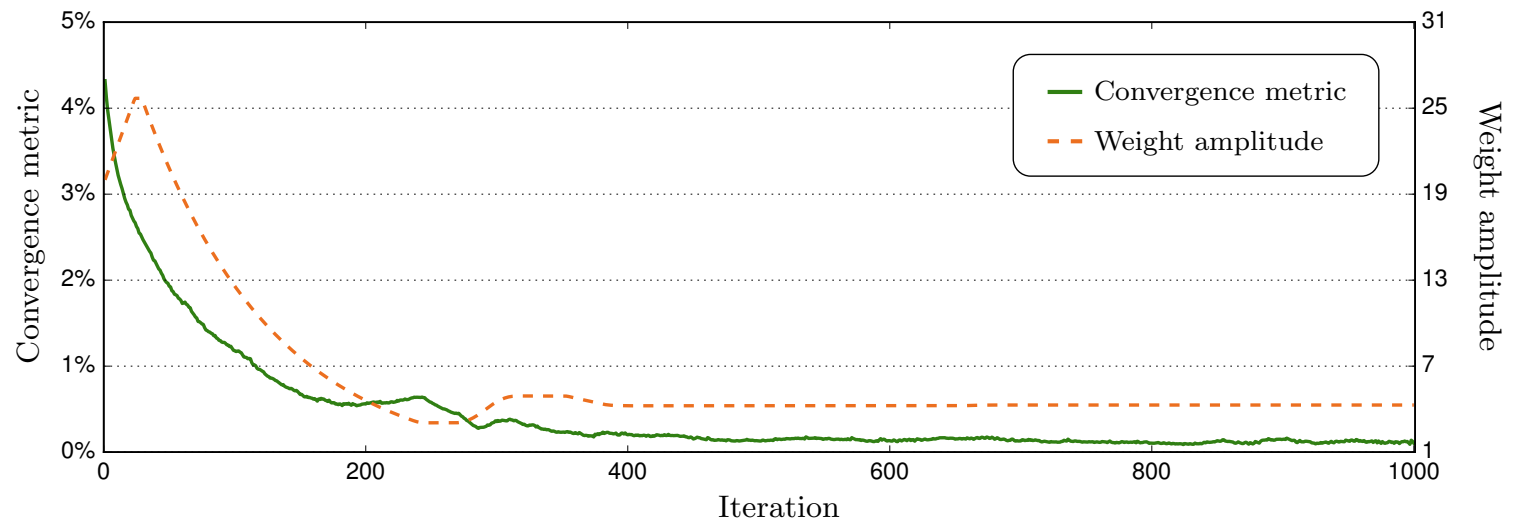

Figure 22: Convergence metric and weight amplitude for the T-junction example.

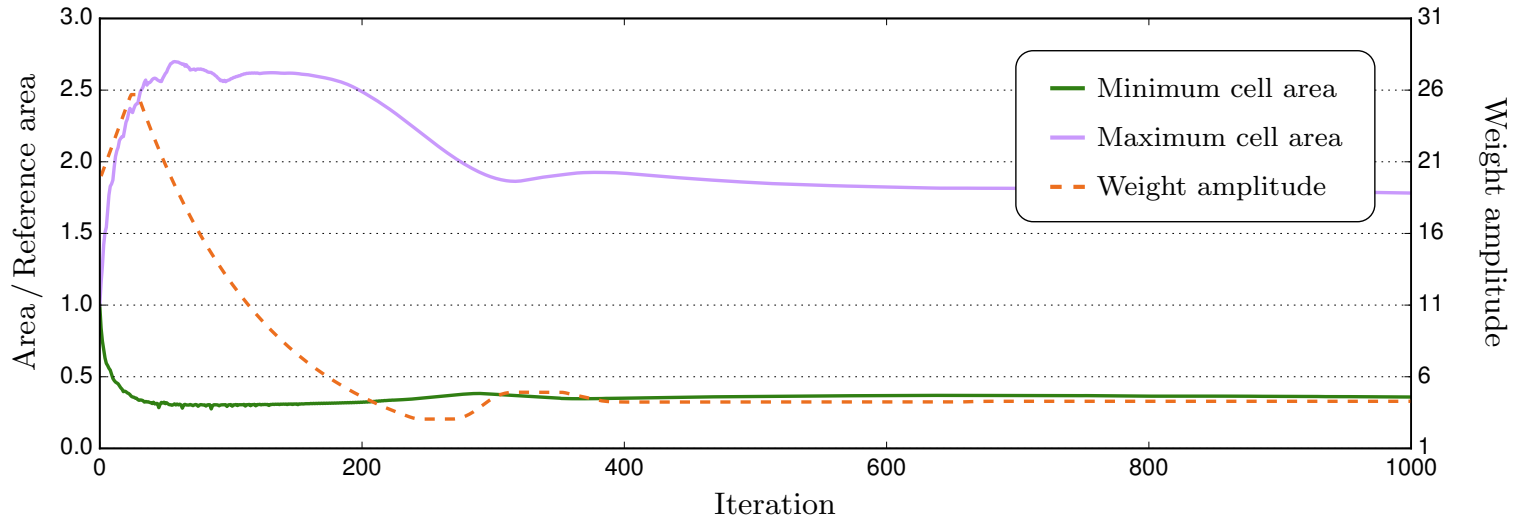

Figure 23: Minimum cell area, maximum cell area, and weight amplitude for the T-junction example. 


\begin{tabular}{cccc}
\hline CFL & $\begin{array}{c}\text { Total number of } \\
\text { time steps }\end{array}$ & $\begin{array}{c}\text { Total number of } \\
\text { relaxation iterations }\end{array}$ & $\begin{array}{c}\text { Average number of relaxation } \\
\text { iterations per time step }\end{array}$ \\
\hline 0.1 & 302 & 934 & 3.09 \\
0.2 & 152 & 874 & 5.75 \\
0.4 & 78 & 804 & 10.31 \\
\hline
\end{tabular}

Table 8: Total number of time steps and mesh relaxation iterations for the dynamic sinusoidal interface.

magnitude is 0.75 , it will take 0.75 time units to reach a magnitude of zero. At that point, the amplitude will increase with a velocity of 1 until the initial amplitude of 0.75 is reached again. The $A^{0}=2$ case of the static sinusoidal after 500 iterations was used as the initial mesh for this example.

For the dynamic portion of the simulation, three different CFL values were used: $0.1,0.2$, and 0.4. The mesh at different time steps for the CFL $=0.4$ case is shown in Figure 24. The mesh relaxation method had no difficulty keeping pace with the change of the sinusoidal's amplitude. Table 8 provides a comparison of the total number of time steps and the total number of relaxation iterations for each CFL number. When the CFL number is increased from 0.1 to 0.4 , the number of required time steps decreases by approximately a factor of 4 , as expected, but the total number of relaxation iterations only decreases by a factor of 1.16. This is because the mesh relaxation needs more iterations per time step to adjust to the larger displacement of the interface. Correspondingly, the average number of relaxation iterations per time step increases by a factor of 3.3 when going from a CFL number of 0.1 to 0.4.

Figure 25 shows the number of relaxation iterations for each time step of the simulation. Also included in the figure is the weight function amplitude at the end of each time step. The figure shows that the weight amplitude changes throughout the simulation. This adjustment is due to the change in the interfacial length. As it gets smaller, a smaller weight function amplitude is needed to maintain the prescribed minimum cell area. The figure also shows that changes in the weight amplitude are usually accompanied by spikes in the relaxation iteration count. This is to be expected, as the relaxation must account for not only the change in interface position, but the change in weight amplitude as well. A history of the minimum and maximum cell area for all three cases is provided in Figure 26. The profile of the minimum area, the maximum area, and the weight amplitude for each case is very similar, suggesting the mesh generated by the relaxation method is independent of the CFL number. Although the maximum cell area changes with the change in weight amplitude, the minimum for all three cases remains close to the target value for the entire simulation, which means the desired resolution is always maintained. 


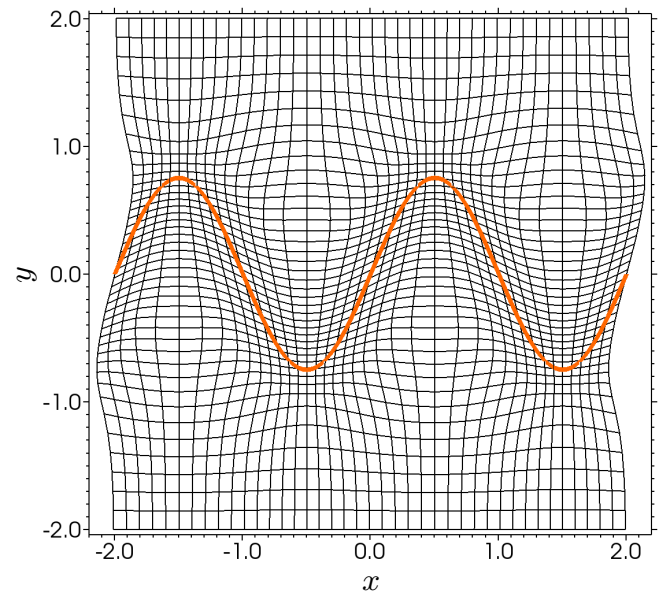

(a) $t=0.0$

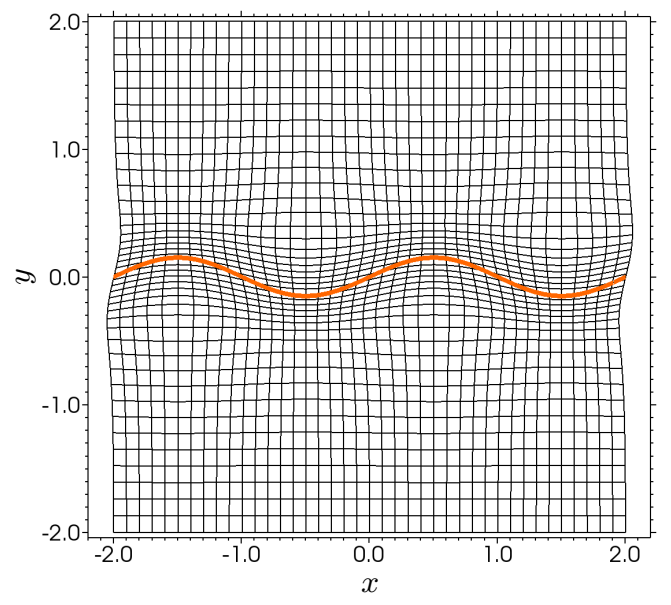

(c) $t=0.6$

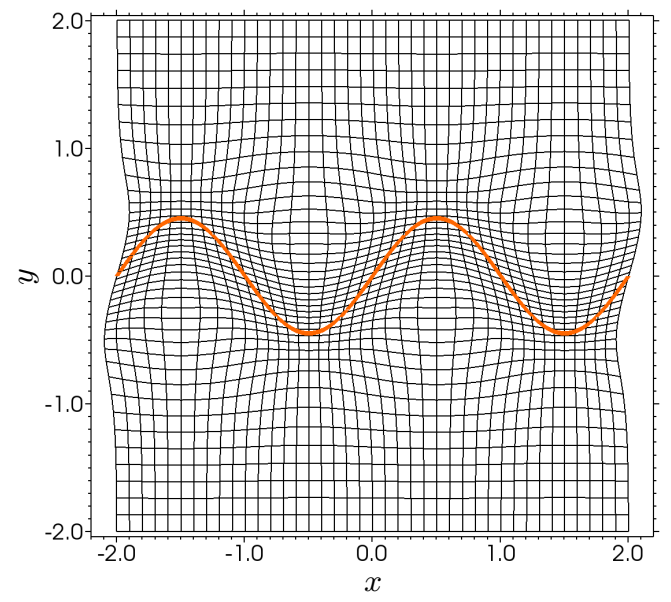

(e) $t=1.2$

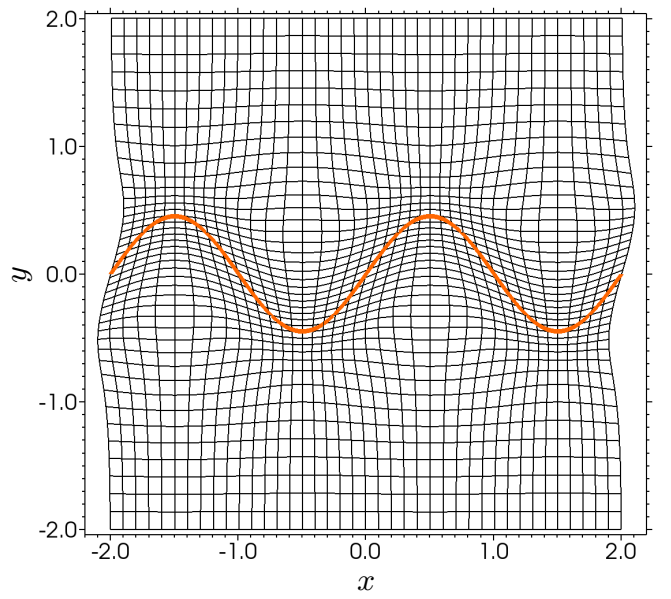

(b) $t=0.3$

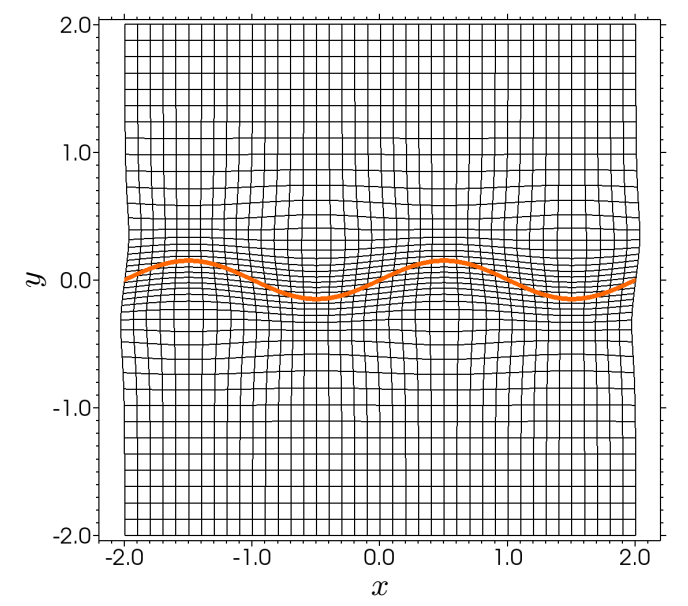

(d) $t=0.9$

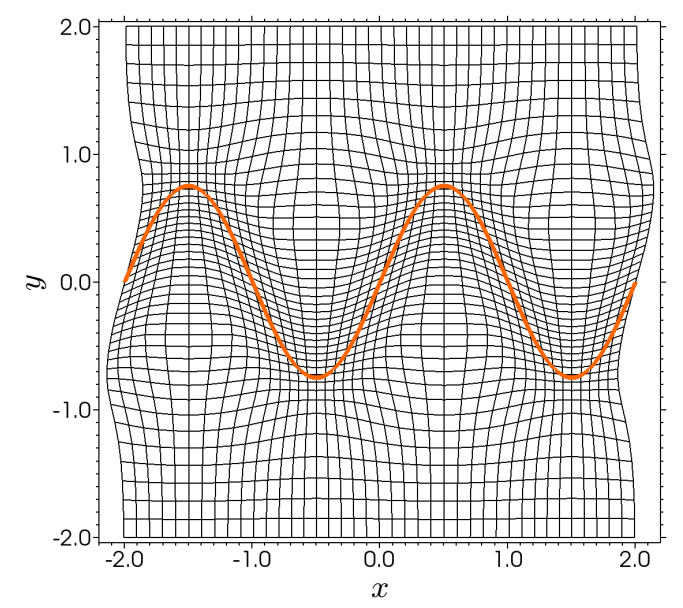

(f) $t=1.5$

Figure 24: Mesh for the dynamic sinusoidal interface. Mesh is shown at various times during CFL $=0.4$ simulation. Interface shown as - 


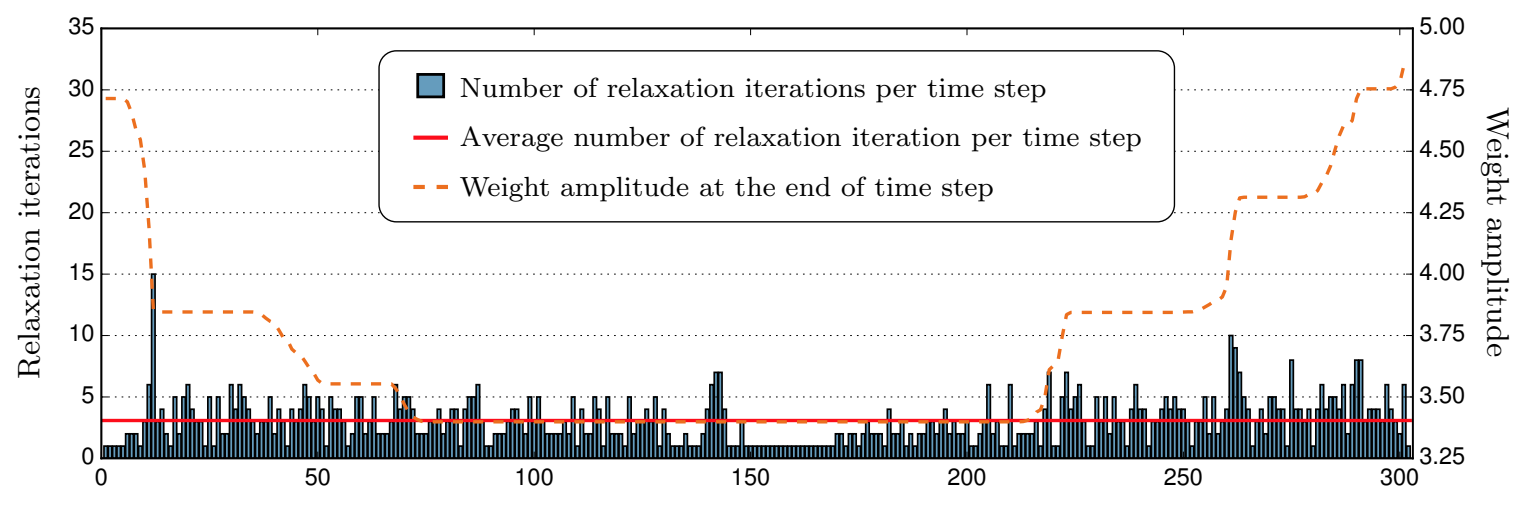

Time step

(a) $\mathrm{CFL}=0.1$ case. Simulation used an average of 3.09 relaxation iterations per time step.

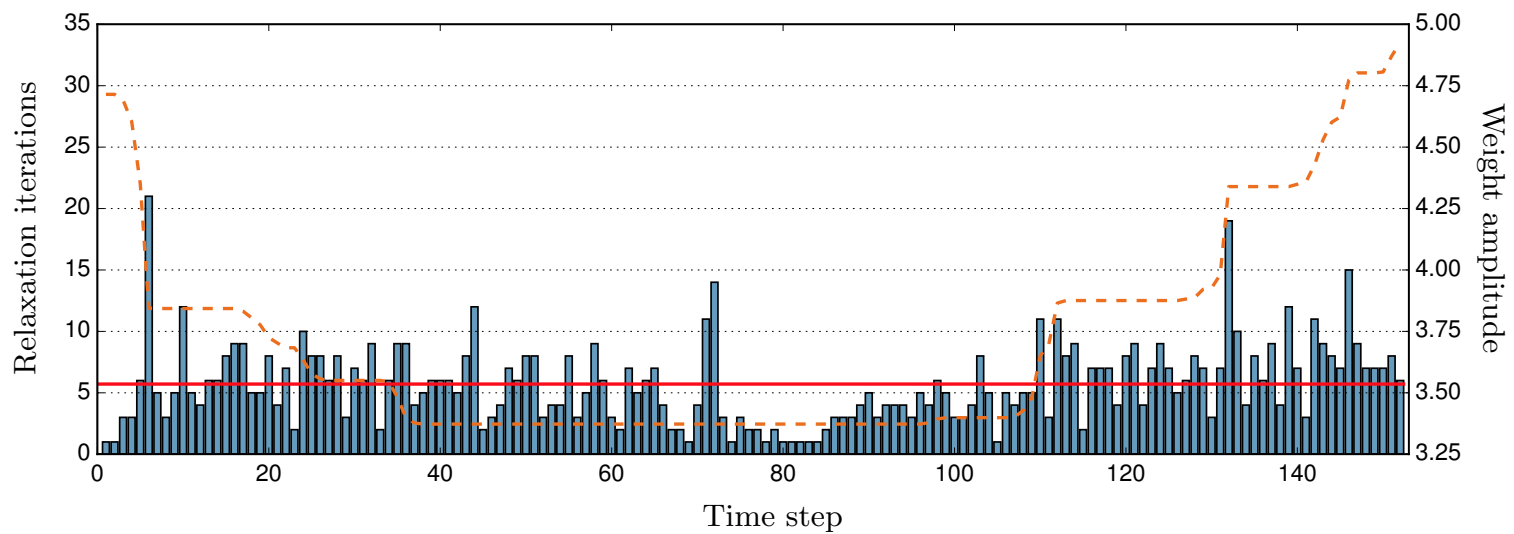

(b) $\mathrm{CFL}=0.2$ case. Simulation used an average of 5.75 relaxation iterations per time step.

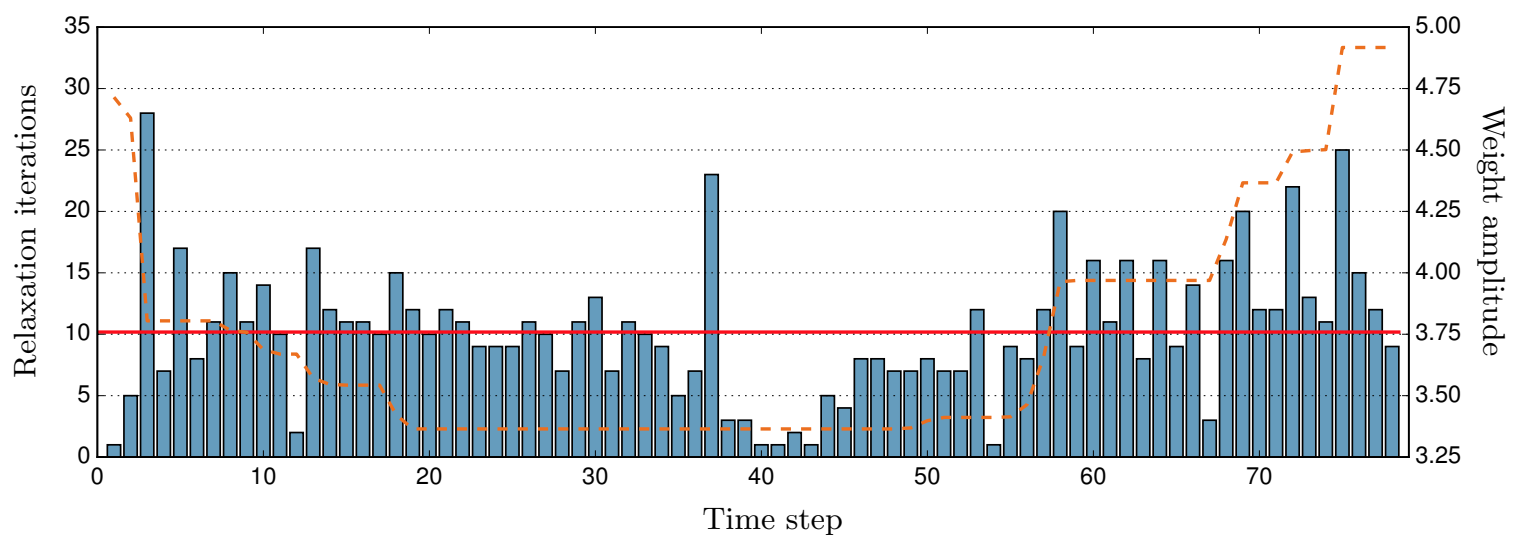

(c) $\mathrm{CFL}=0.4$ case. Simulation used an average of 10.31 relaxation iterations per time step.

Figure 25: Number of relaxation iterations per time step for the dynamic sinusoidal interface. 


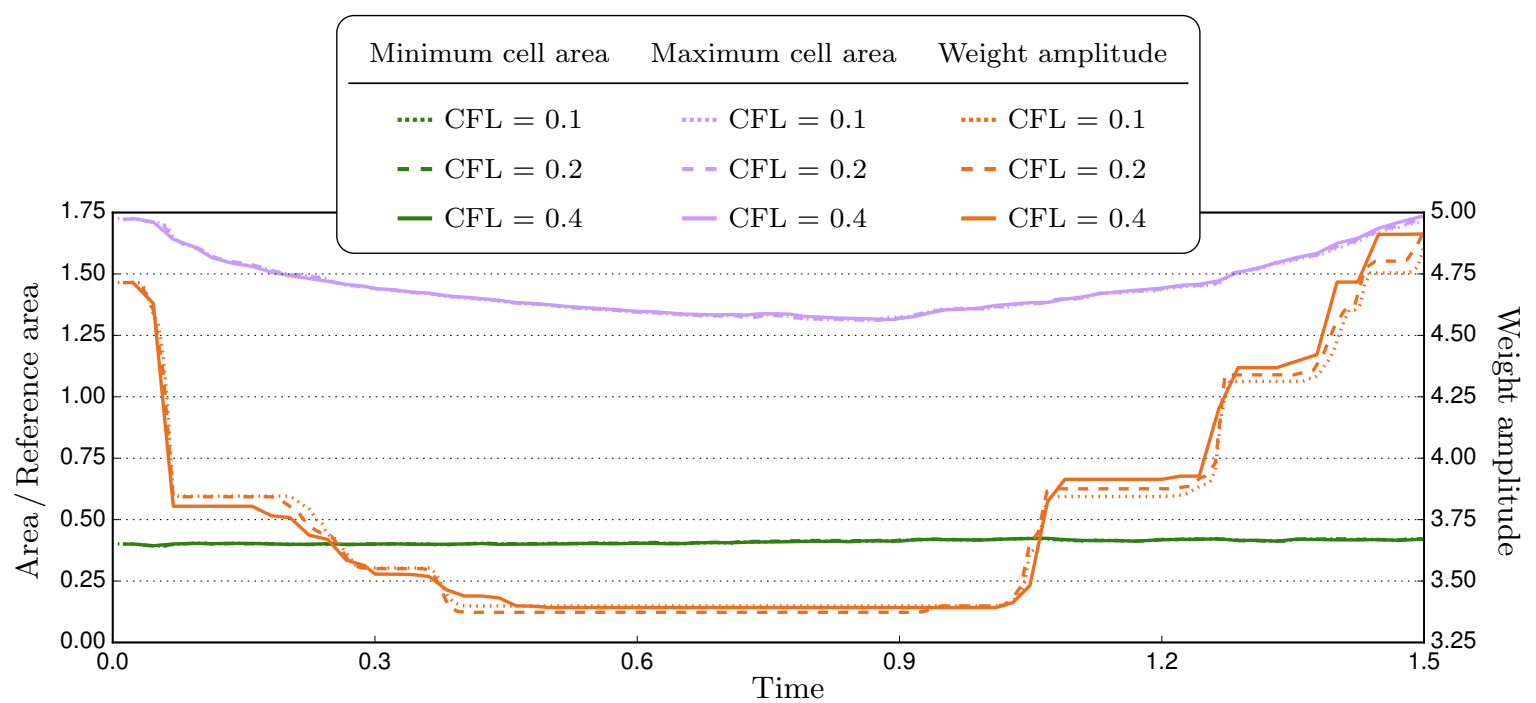

Figure 26: Minimum cell area, maximum cell area, and weight amplitude for the dynamic sinusoidal interface.

\subsection{Cruciform}

The second dynamic interface example is for a rotating cruciform. The initial geometry of the interface and weight function parameters are identical to the static cruciform. The dynamic simulation will cover one full counterclockwise rotation of the cruciform with a non-dimensional period of 1 . The mesh from the $A^{0}=15$ case after 2000 iterations was used as the initial mesh. Unlike the dynamic sinusoidal case, the initial mesh has not reached a final weight amplitude yet. This is done intentionally to demonstrate how the mesh and the weight function amplitude can continue to adjust during the evolution of the interface.

The same three CFL values were used for the dynamic portion of this simulation. The mesh at different time steps for the CFL $=0.4$ case is shown in Figure 27. Similarly to the sinusoidal case, the mesh was able to maintain the desired mesh resolution around the cruciform as it rotated. The total number of time steps and total number of relaxation iterations for this case are shown in Table 9. The ratios for the total number of time steps, total number of relaxation iterations, and average relaxation iterations for the CFL $=0.1$ and $\mathrm{CFL}=0.4$ simulations are very similar to the dynamic sinusoidal example.

Figure 28 shows the number of relaxation iterations for each time step of the CFL $=0.4$ case. The figure shows that the weight function amplitude was able to adjust and eventually converged to its final value during the dynamic portion of the simulation. Just as in the sinusoidal example, a change in the weight function amplitude usually came with a spike in the number of relaxation iterations. The low-frequency oscillation seen in the number of iterations is related to the alignment of the cruciform with the mesh. The minimums correspond to the cruciform being aligned with $x$ and $y$-axis, while the peaks are when the cruciform is at an angle of $45^{\circ}$ with the $x$-axis. The minimum and maximum cell area at the end of each time step is depicted in Figure 29. The plot shows that despite the weight amplitude needing to adjust 


\begin{tabular}{cccc}
\hline CFL & $\begin{array}{c}\text { Total number of } \\
\text { time steps }\end{array}$ & $\begin{array}{c}\text { Total number of } \\
\text { relaxation iterations }\end{array}$ & $\begin{array}{c}\text { Average number of relaxation } \\
\text { iterations per time step }\end{array}$ \\
\hline 0.1 & 1612 & 7599 & 4.71 \\
0.2 & 808 & 7337 & 9.08 \\
0.4 & 406 & 6965 & 17.16 \\
\hline
\end{tabular}

Table 9: Total number of time steps and mesh relaxation iterations for the dynamic cruciform interface.

during the initial portion of the simulation, the minimum area remains very close to the target value of $0.25 \Omega_{R}$ for the entire simulation. The profiles for the other two CFL values are very similar.

\section{Conclusion}

In this work, we presented a new DG based weighted condition number mesh relaxation method that can cluster grid cells near dynamically evolving interfaces. The weight function is computed from the interface's level set function and is represented as an $\operatorname{rDG}\left(\mathrm{P}_{1} \mathrm{P}_{2}\right)$ reconstruction with WENO used to smooth in-cell discontinuities. The DG projection allows us to readily compute the derivatives required for the condition number optimization. In many legacy codes, a level set representation of interfaces may not be available. For these cases, we developed a method for generating a low-order level set function from an interface prescribed by discrete cell-centered values (denoted as an index function in this work). The low-order level set is expressed as an $\mathrm{rDG}\left(\mathrm{P}_{0} \mathrm{P}_{2}\right)$ reconstruction and is used to compute the weight function in the same manner as the actual level set. Meshes for a number of example static geometries were presented. The examples were chosen to test the method on complex geometries with discontinuities in the level set function and on interfaces for multiple materials. For all the cases, the method was able to refine the mesh around the interfaces and obtain the desired mesh resolution.

Since the goal of this work is to provide a new mesh relaxation method for ALE simulations, two dynamic interface examples were presented. For both cases, the interface was defined from an index function. The interfaces were moved with a prescribed motion and a fixed CFL number. At each time step, the mesh was allowed to relax until a desired level of convergence was obtained. For both examples, the mesh was able to keep pace with the moving interface and maintain the desired level of resolution with only a few relaxation iterations per time step, thus demonstrating the potential of the method for practical ALE simulations. While the original condition number mesh relaxation used in many ALE methods provides very good mesh smoothing and prevents mesh tangling, it is unable to maintain the fidelity of the mesh near dynamically evolving multi-material interfaces. The method presented in this work provides an approach for maintaining a desired resolution near a dynamically moving interface, while also retaining the mesh smoothing capabilities 


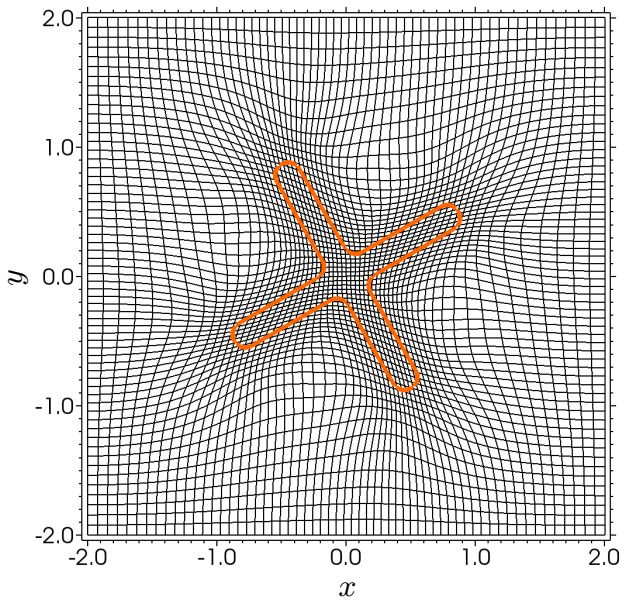

(a) $t=0.0$

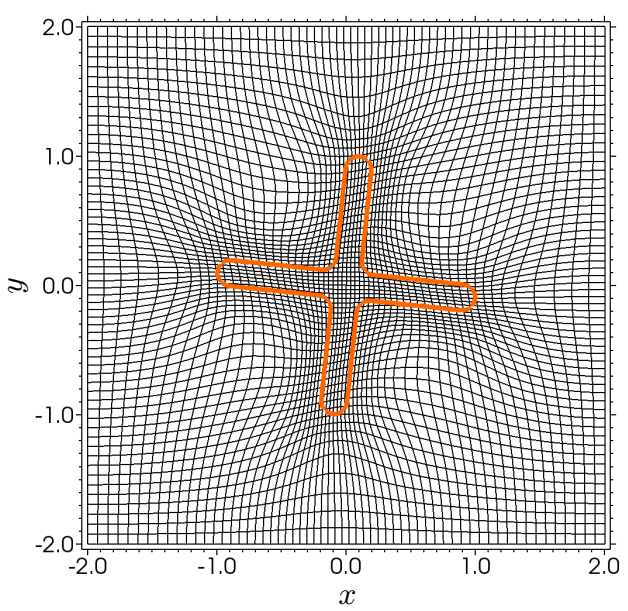

(c) $t=0.4$

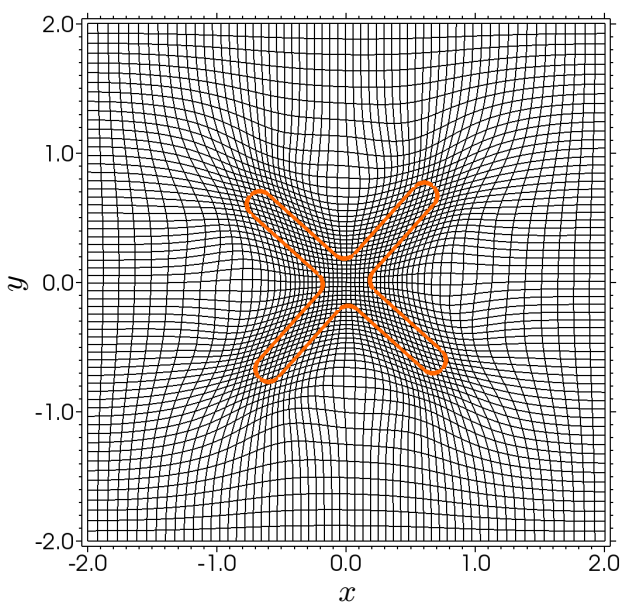

(e) $t=0.8$

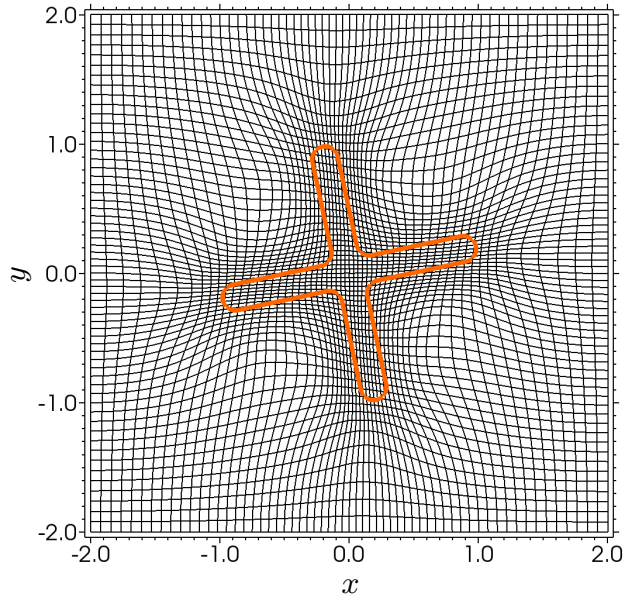

(b) $t=0.2$

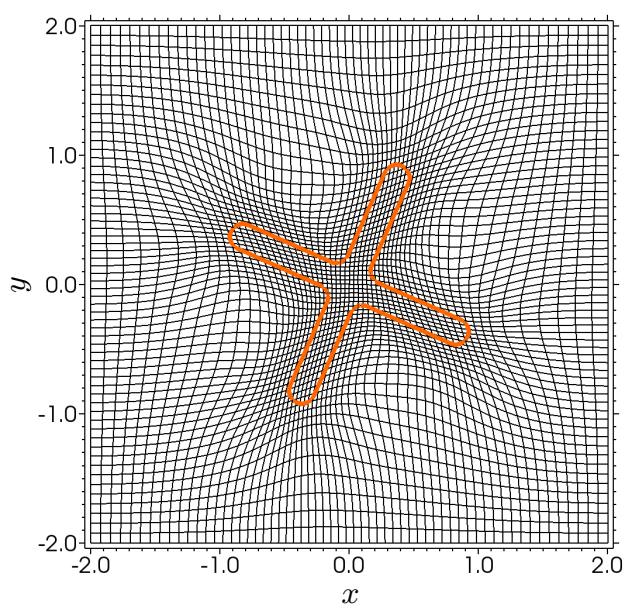

(d) $t=0.6$

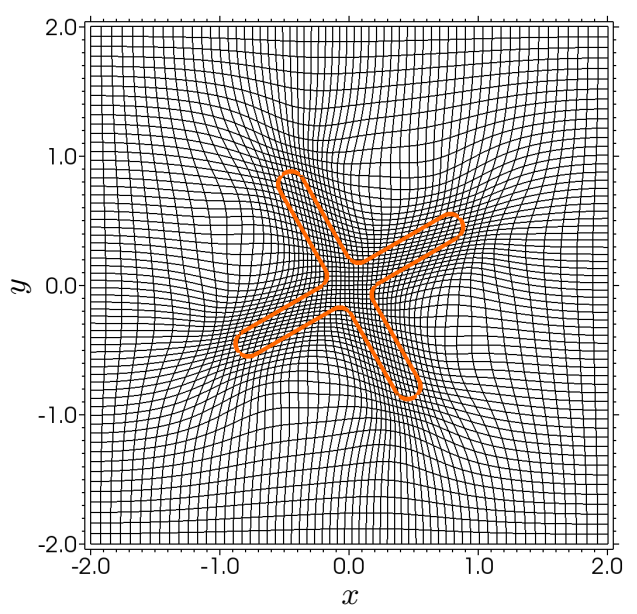

(f) $t=1.0$

Figure 27: Mesh for the dynamic cruciform interface. Mesh is shown at various times during CFL $=0.4$ simulation. Interface shown as 


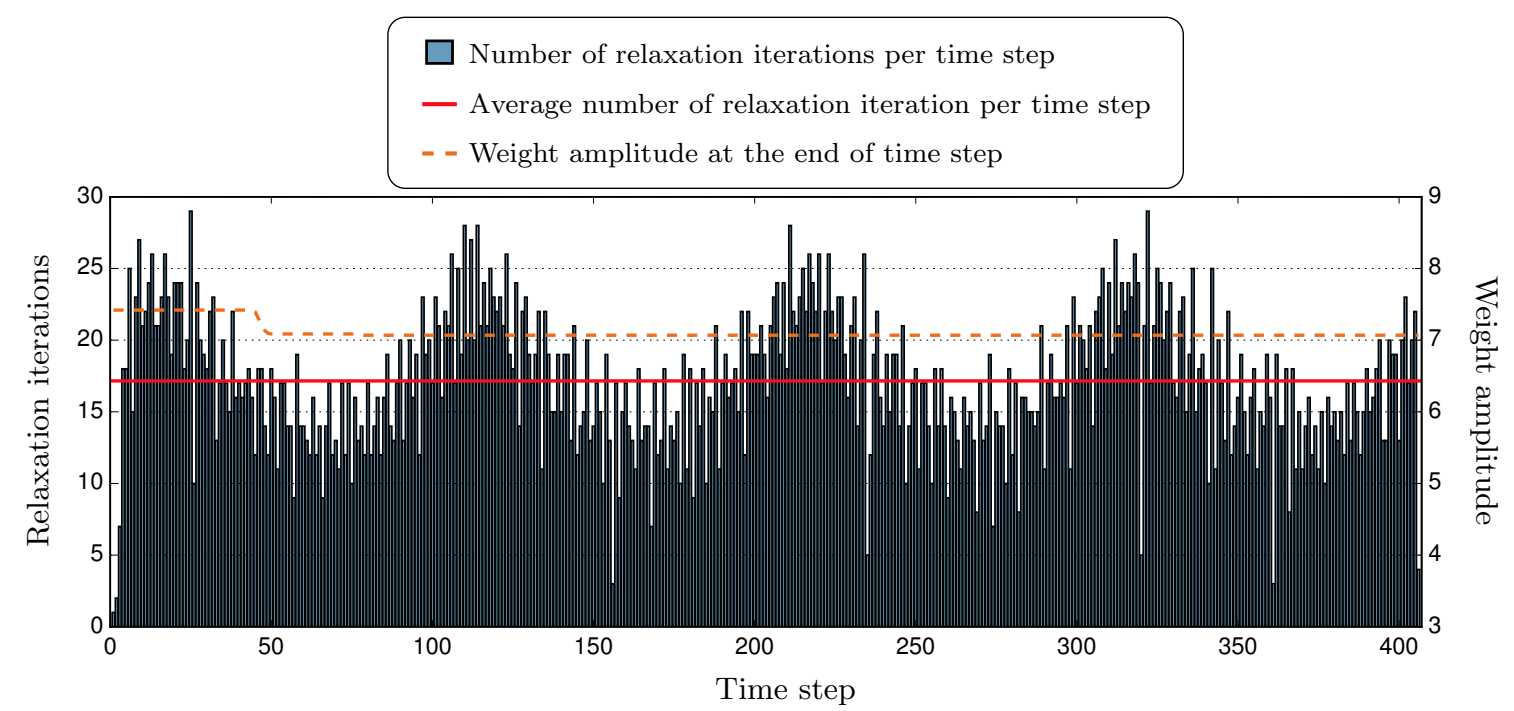

Figure 28: Number of relaxation iterations per time step for the dynamic cruciform interface with CFL $=0.4$. Simulation used an average of 17.16 relaxation iterations per time step.

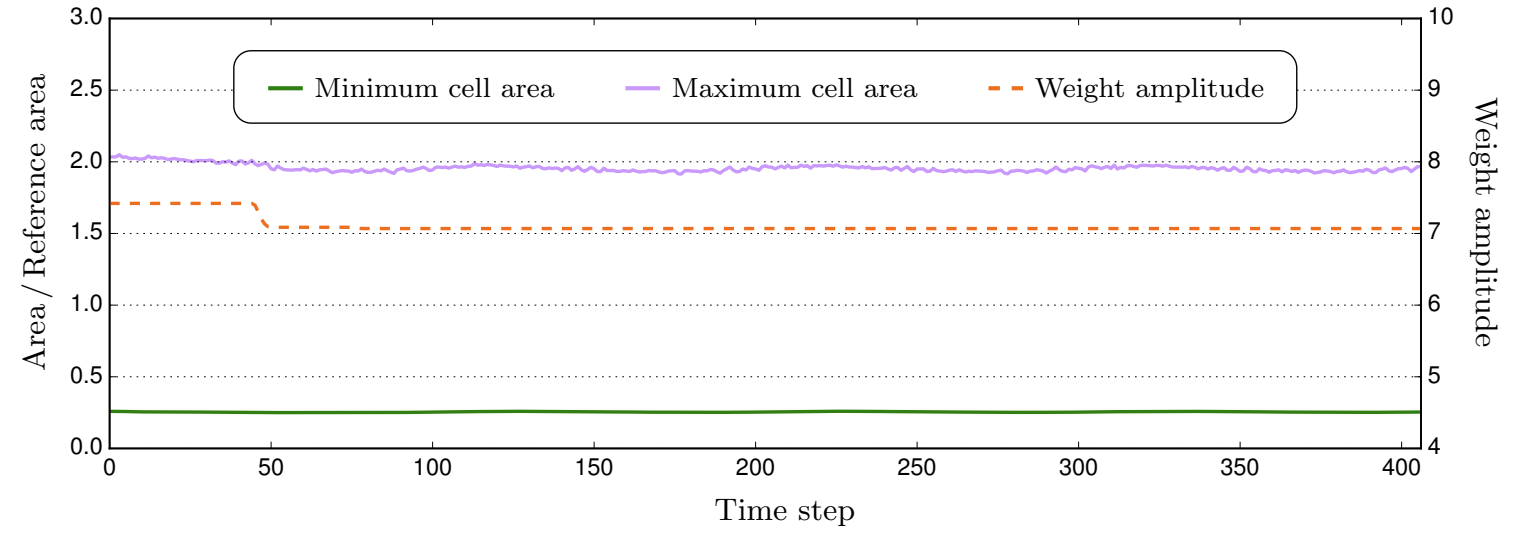

Figure 29: Minimum cell area, maximum cell area, and weight amplitude for the dynamic cruciform interface with CFL $=0.4$. 
of the original condition number mesh relaxation algorithm.

\section{Acknowledgments}

This work was performed under the auspices of the U.S. Department of Energy by Lawrence Livermore National Laboratory under Contract DE-AC52-07NA27344.

\section{References}

[1] C. W. Hirt, A. A. Amsden, J. L. Cook, Arbitrary Lagrangian-Eulerian computing method for all flow speeds, Journal of Computational Physics 14 (1974) 227-253. doi:10.1016/0021-9991(74)90051-5.

[2] P. M. Knupp, L. G. Margolin, M. Shashkov, Reference Jacobian optimization-based rezone strategies for arbitrary Lagrangian Eulerian methods, Journal of Computational Physics 176 (2002) 93 - 128. doi:10.1006/jcph.2001.6969.

[3] A. M. Winslow, Equipotential zoning of two-dimensional meshes, Technical Report UCRL-7312, Lawrence Radiation Laboratory, 1963.

[4] P. M. Knupp, Achieving finite element mesh quality via optimization of the Jacobian matrix norm and associated quantities. Part I - a framework for surface mesh optimization, International Journal for Numerical Methods in Engineering 48 (2000) 401-420. doi:10.1002/(SICI) 1097-0207 (20000530) 48:3<401 : : AID-NME880>3 . 0 . C0; 2-D.

[5] P. M. Knupp, Achieving finite element mesh quality via optimization of the Jacobian matrix norm and associated quantities. Part II - a framework for volume mesh optimization and the condition number of the Jacobian matrix, International Journal for Numerical Methods in Engineering 48 (2000) 1165-1185. doi:10.1002/(SICI) 1097-0207 (20000720)48: 8<1165: : AID-NME940>3.0.CO;2-Y.

[6] P. M. Knupp, N. Robidoux, A framework for variational grid generation: Conditioning the Jacobian matrix with matrix norms, SIAM Journal on Scientific Computing 21 (2000) 2029-2047. doi:10.1137/S1064827598341633.

[7] P. Váchal, P.-H. Maire, Discretizations for weighted condition number smoothing on general unstructured meshes, Computers \& Fluids 46 (2011) 479 - 485. doi:10.1016/j.compfluid.2010.10.025

[8] S. P. Schofield, Weighted condition number relaxation with discrete weights, Technical Report LLNL-PROC-666100, Lawrence Livermore National Laboratory, 2014.

[9] H. Luo, J. D. Baum, R. Löhner, A discontinuous Galerkin method based on a Taylor basis for the compressible flows on arbitrary grids, Journal of Computational Physics 227 (2008) 8875 - 8893. doi:10.1016/j.jcp.2008.06.035.

[10] H. Luo, Y. Xia, S. Li, R. Nourgaliev, C. Cai, A Hermite WENO reconstruction-based discontinuous Galerkin method for the Euler equations on tetrahedral grids, Journal of Computational Physics 231 (2012) 5489 - 5503. doi:10.1016/j.jcp. 2012.05.011.

[11] H. Luo, Y. Xia, S. Spiegel, R. Nourgaliev, Z. Jiang, A reconstructed discontinuous Galerkin method based on a hierarchical WENO reconstruction for compressible flows on tetrahedral grids, Journal of Computational Physics 236 (2013) 477 492. doi:10.1016/j.jcp.2012.11.026.

[12] M. Dumbser, D. S. Balsara, E. F. Toro, C.-D. Munz, A unified framework for the construction of one-step finite volume and discontinuous Galerkin schemes on unstructured meshes, Journal of Computational Physics 227 (2008) 8209 - 8253. doi:10.1016/j.jcp. 2008.05.025.

[13] M. Dumbser, Arbitrary high order $P_{N} P_{M}$ schemes on unstructured meshes for the compressible Navier-Stokes equations, Computers \& Fluids 39 (2010) 60 - 76. doi:10.1016/j. compfluid.2009.07.003. 
[14] P. T. Greene, S. P. Schofield, R. Nourgaliev, Dynamic mesh adaptation for front evolution using discontinuous Galerkin based weighted condition number mesh relaxation, Technical Report LLNL-TN-695525, Lawrence Livermore National Laboratory, 2016.

[15] M. Sussman, P. Smereka, S. Osher, A level set approach for computing solutions to incompressible two-phase flow, Journal of Computational Physics 114 (1994) 146 - 159. doi:10.1006/jcph.1994.1155.

[16] J. A. Sethian, A fast marching level set method for monotonically advancing fronts, Proceedings of the National Academy of Sciences 93 (1996) 1591-1595.

[17] R. Nourgaliev, P. T. Greene, S. P. Schofield, Marker re-distancing (MRD) algorithm for high-fidelity interface tracking on arbitrary meshes, Journal of Computational Physics (Manuscript in Preparation).

[18] C. Hirt, B. Nichols, Volume of fluid (VOF) method for the dynamics of free boundaries, Journal of Computational Physics 39 (1981) 201 - 225. doi:10.1016/0021-9991(81)90145-5.

[19] D. Adalsteinsson, J. A. Sethian, A fast level set method for propagating interfaces, Journal of Computational Physics 118 (1995) 269 - 277. doi:10.1006/jcph.1995.1098.

[20] R. D. Hornung, J. A. Keasler, The RAJA portability layer: Overview and status, Technical Report LLNL-TR-661403, Lawrence Livermore National Laboratory, 2014. 